\title{
HST/ACS observations of the old and metal-poor Sagittarius dwarf irregular galaxy ${ }^{\star}$
}

\author{
Y. Momany ${ }^{1}$, E. V. Held ${ }^{2}$, I. Saviane ${ }^{3}$, L. R. Bedin ${ }^{1,4}$, M. Gullieuszik ${ }^{1,2}$, M. Clemens ${ }^{2}$, \\ L. Rizzi ${ }^{5}$, M. R. Rich ${ }^{6}$, and K. Kuijken ${ }^{7}$
}

1 Dipartimento di Astronomia, Università di Padova, Vicolo dell'Osservatorio 2, 35122 Padova, Italy e-mail: momany@pd.astro.it

2 Osservatorio Astronomico di Padova, Vicolo dell'Osservatorio 5, 35122 Padova, Italy

3 European Southern Observatory, 3107 Alonso de Cordova, Vitacura, Casilla 19001, Santiago 19, Chile

${ }^{4}$ European Southern Observatory, Karl-Schwarzschild-Str. 2, 85748 Garching b. München, Germany

5 Institute for Astronomy, 2680 Woodlawn Drive, Honolulu, USA

${ }^{6}$ Department of Physics and Astronomy, UCLA, 430 Portola Plaza, Los Angeles CA, 90095-1547, USA

7 Leiden Observatory, PO Box 9513, 2300 RA Leiden, The Netherlands

Received 22 January 2005 / Accepted 27 April 2005

\begin{abstract}
We have obtained deep color-magnitude diagrams (CMDs) of the Sagittarius dwarf irregular galaxy with the Advanced Camera for Surveys (ACS) on board the Hubble Space Telescope. The new diagrams show for the first time the star formation history of SagDIG. The young main-sequence is traced down to $m_{F 606 W} \simeq 27.5$, well separated from the older He burning blue loop stars. The wide color range spanned by the blue and red super-giants is consistent with Padua evolutionary models with $Z \sim 0.0004$. A well-defined red giant branch indicates that the galaxy is dominated by a stellar population older than $1 \mathrm{Gyr}$, for which we have estimated a metallicity in the range $[\mathrm{Fe} / \mathrm{H}]=-2.2$ to -1.9 depending on the adopted reddening. The identification of several $\mathrm{C}$ stars on a luminous asymptotic giant branch, as well as the presence of a conspicuous red clump with an elongated structure, are indicative of an extended star formation epoch between 1 and 10 Gyr ago. Perhaps most importantly, we have identified a small population of stars on a genuinely old red horizontal branch at $m_{F 606 W}=25.70 \pm 0.05$, which sets the first epoch of star formation in SagDIG back to 9-10 Gyr ago. This shows that SagDIG has been able to maintain a very low mean metallicity over a $10 \mathrm{Gyr}$ life time, and that all Local Group dwarf galaxies share an ancient ( $\geq 10 \mathrm{Gyr})$ initial episode of star formation. Thus, at the moment, IZw 18 remains the only exception to this general trend, favoring the so-called "young galaxy" scenario where a very low metallicity galaxy is not necessarily old. However, a re-analysis of ACS observations of IZw 18 suggests that an older RGB population may be present also in IZw 18.

In order to address the interplay between stars and Inter-Stellar Medium, we compare the distribution of atomic hydrogen with that of stellar populations of various ages. The youngest stars are found in clumps located: (i) near to, but not coincident with, the highest column densities of H I, and (ii) in tails reminiscent of propagating star formation. We find, however, little correlation between stellar populations older than $\sim 1$ Gyr and the H I. In particular we fail to find any generation of stars that preferentially lie within the large $\mathrm{H}$ I hole. We therefore exclude the possibility that the ring-like H I distribution is the result of multiple supernova events. Alternative scenarios have to be explored for the ring-like H I morphology of SagDIG, which amongst dwarf irregular galaxies, is not unusual.
\end{abstract}

Key words. galaxies: individual: SagDIG - galaxies: individual: IZw 18 - ISM: structure - galaxies: dwarf Hertzprung-Russell (HR) and C-M diagrams - stars: Population II

\section{Introduction}

The Hubble Space Telescope has made it possible to resolve the stellar populations of dwarf irregular galaxies (dIrr) into individual stars both in the Local Group (e.g. Dohm-Palmer et al. 1997, 1998, 2002; Tolstoy et al. 1998; Dolphin 2000;

* Based on observations with the NASA/ESA Hubble Space Telescope, (GO 9820) obtained at the Space Telescope Science Institute, which is operated by AURA, Inc., under NASA contract NAS 5-26555.
Dolphin et al. 2001, 2002, 2003; Skillman et al. 2003) and beyond (as in the case of the blue-compact dwarf IZw 18 at a distance between 10-15 Mpc; Aloisi et al. 1999; Izotov \& Thuan 2004). In all cases, color-magnitude diagrams (CMD) can be constructed to estimate their distance and metallicity and constrain the recent star formation history (SFH) of the systems. In the nearest galaxies, a wealth of evolutionary details can be observed, otherwise hampered by poor resolution in groundbased studies. 
The quest for very metal-poor galaxies among dwarf irregulars started soon after the Searle and Sargent (1972) discovery that the interstellar medium (ISM) of I Zw 18 has an oxygen abundance that is $\sim 1 / 50$ of the solar one. As Searle \& Sargent pointed out, this is compatible with two scenarios: either the galaxy is now experiencing its first star-formation (SF) episode, or, if it contains $\sim 10$ Gyr old stars, its SF must have proceeded in strong bursts separated by quiescent phases. The first hypothesis (a young galaxy) is relevant in the context of cosmological models that assume hierarchical formation of structures in a cold dark matter dominated Universe. In that scenario, dwarf dark matter halos $\left(\sim 10^{8} M_{\odot}\right)$ would be the first objects to condense out of the Hubble flow, and it is therefore important to understand whether they already contained stars before merging into larger galaxies. In fact, IZw 18 is a difficult object to study because of its distance. We may ask ourselves an equivalent, possibly easier to answer, question: are there any galaxies with a very metal-poor young population and, at the same time, $a \sim 10$ Gyr old generation of stars? The existence of such old, metal-poor stellar systems would imply that very low metal abundances are not inconsistent with an ancient epoch of galaxy formation. In order to answer this question, we have undertaken an HST/ACS study the Sagittarius dwarf irregular galaxy (also known as SagDIG or UKS 1927-177). The interest of SagDIG arises from the presence of on-going star formation in an extremely metal-poor environment. The fact that SagDIG is a nearby $(\simeq 1.1 \mathrm{Mpc})$ galaxy makes it an ideal target to test locally the young galaxy hypothesis.

The most recent photometric studies of SagDIG are those of Karachentsev et al. (1999), Lee \& Kim (2000), and Momany et al. (2002, hereafter M02). Lee \& Kim and Karachentsev and coll. concluded that SagDIG has the lowest stellar metallicity (up to $[\mathrm{Fe} / \mathrm{H}] \sim-2.45$ ) among Local Group star-forming galaxies. M02 presented deep BVI photometry of SagDIG obtained from ESO NTT data (we refer the reader to that paper for a full summary of previous work). In M02 a differential reddening scenario was suggested to explain the relatively red color of main-sequence stars. Our hypothesis was that some internal reddening (in addition to the foreground reddening) affects the young stars in star-forming regions. The revised metallicity, $[\mathrm{Fe} / \mathrm{H}]=-2.1 \pm 0.2$, turned out to be in better agreement with the general trend of the (stellar) metallicity-luminosity relation for dwarf irregular galaxies, indicating a "normal", although metal-poor, dIrr galaxy.

The cold and warm ISM of SagDIG and its kinematics have been the subject of several investigations. Skillman et al. (1989) obtained optical spectrophotometry of the most luminous $\mathrm{H}$ II regions, and estimated a $[\mathrm{O} / \mathrm{H}]$ abundance $\sim 3 \%$ of the solar value, a result independently confirmed by the new measurements of Saviane et al. (2002).

High-resolution, high sensitivity VLA observations of the SagDIG H I content have been obtained by Young \& Lo (1997) (see also Lo et al. 1993). About $1.2 \times 10^{7} M_{\odot}$ of $(\mathrm{H}+\mathrm{He})$ have been estimated by M02 using $(m-M)_{0}=25.14$ and the H I column density given by Young \& Lo. SagDIG thus appears to have a relatively high mass fraction in the form of neutral gas, $M_{\mathrm{HI}} / L_{B}=1.6$, a value typical of the H I content in dwarf irregular galaxies (see Mateo 1998). The H I is distributed in an almost symmetric ring which was interpreted as produced by the combined effects of stellar winds and supernovae.

This paper presents our new HST/ACS observations, clearly overcoming the limitations of previous ground-based observations and probing the young, intermediate age, and old stellar populations in SagDIG. The results of those observations are presented in this paper. The paper is organized as follows. In Sect. 2 we introduce our HST/ACS observations and data reduction. Section 3 presents the ACS color-magnitude diagrams and their main features. In Sect. 4 we re-derive the distance to SagDIG, the mean metallicity of its red giants stars, and, most importantly, provide evidence for a $10 \mathrm{Gyr}$ old horizontal branch population. The recent star formation is then analyzed in Sect. 5. Section 6 compares the spatial distribution of the stars and the $\mathrm{HI}$ and discusses the relationship between the composite stellar populations of SagDIG and the morphology of its ISM. The principal results of our ACS study are finally summarized in Sect. 7.

\section{Observations and data reduction}

Images of SagDIG were taken using the ACS on board the HST, in the course of one visit (on August 18, 2003; program GO 9820) for a total of 3 orbits. The observed field covers the main body of the galaxy and is centered at $l=21^{\circ} .06$, $b=-16.28$. Through the ACS-Wide Field Channel (WFC), we obtained $5 \times 396 \mathrm{~s}$ images in the $F 606 \mathrm{~W}$ filter, $5 \times 419 \mathrm{~s}$ in $F 475 \mathrm{~W}$ and $5 \times 421 \mathrm{~s}$ in $F 814 \mathrm{~W}$. The series of 5 images in $F 475 \mathrm{~W}$ and $F 606 \mathrm{~W}$ were dithered so as to allow filling the gap between the CCDs, removal of cosmic rays, and optimization of the point spread function (PSF) and spatial sampling (Anderson \& King 2000). No attention has been paid in the recovering the gap in $F 814 \mathrm{~W}$ images (sub-arcsec dithering pattern), so as to avoid residuals in the geometric distortion correction when second epoch $F 814 \mathrm{~W}$ images (to be obtained in cycle 14) are used to derive the proper motion of observed stars.

The instrument distortion was corrected using ACS/WFC geometrical distortion correction maps made by Anderson (2004, priv. comm.). All images in the same filter were then stacked to build a median image, with pixel oversampling by a factor of 2. These steps allowed us to improve the signal-tonoise ratio and the PSF sampling. Using these images, standard DAOPHOT/ALLSTAR (Stetson 1987) routines were employed to derive both the PSF magnitudes and their aperture corrections. A quadratically variable PSF was adopted to account for the PSF variations across the field of ACS (Krist 2003). The photometric calibration of the ACS/WFC filters follows Bedin et al. (2004). This zero-point photometric calibration is based on the Vega spectrum and follows the recipes given by Holtzmann et al. (1995) for WFPC2, using the most updated in-flight characterization of the camera (Sirianni et al. 2004).

Photometric errors and completeness were estimated from extensive artificial stars experiments following procedures similar to those of M02. More than 50000 stars were added to the stacked images in a range of magnitudes and colors matching the distribution of SagDIG stars in the observed color-magnitude diagrams. The artificial stars were placed in 
Table 1. Photometric errors and completeness as derived from artificial star experiments.

\begin{tabular}{lcccccc}
\hline \hline mag & $\sigma_{F 475 W}$ & $C_{F 475 W}$ & $\sigma_{F 606 W}$ & $C_{F 606 W}$ & $\sigma_{F 814 W}$ & $C_{F 814 W}$ \\
\hline 20.00 & 0.010 & 1.000 & 0.010 & 1.000 & 0.010 & 1.000 \\
20.40 & 0.010 & 1.000 & 0.010 & 1.000 & 0.010 & 1.000 \\
20.80 & 0.010 & 1.000 & 0.010 & 1.000 & 0.009 & 0.999 \\
21.20 & 0.010 & 1.000 & 0.010 & 1.000 & 0.009 & 0.999 \\
21.60 & 0.010 & 1.000 & 0.010 & 1.000 & 0.011 & 0.999 \\
22.00 & 0.010 & 1.000 & 0.010 & 1.000 & 0.013 & 0.997 \\
22.40 & 0.010 & 1.000 & 0.008 & 1.000 & 0.013 & 0.996 \\
22.80 & 0.010 & 1.000 & 0.012 & 0.999 & 0.016 & 0.996 \\
23.20 & 0.009 & 1.000 & 0.014 & 1.000 & 0.021 & 0.994 \\
23.60 & 0.012 & 1.000 & 0.016 & 0.999 & 0.025 & 0.991 \\
24.00 & 0.019 & 1.000 & 0.019 & 0.997 & 0.033 & 0.988 \\
24.40 & 0.027 & 0.996 & 0.023 & 0.996 & 0.043 & 0.989 \\
24.80 & 0.036 & 0.993 & 0.028 & 0.994 & 0.052 & 0.990 \\
25.20 & 0.046 & 0.989 & 0.036 & 0.992 & 0.066 & 0.963 \\
25.60 & 0.060 & 0.988 & 0.047 & 0.991 & 0.086 & 0.894 \\
26.00 & 0.077 & 0.974 & 0.059 & 0.988 & 0.114 & 0.801 \\
26.40 & 0.097 & 0.932 & 0.076 & 0.978 & 0.148 & 0.715 \\
26.80 & 0.125 & 0.807 & 0.102 & 0.966 & 0.186 & 0.603 \\
27.20 & 0.167 & 0.677 & 0.137 & 0.924 & 0.225 & 0.384 \\
27.60 & 0.211 & 0.428 & 0.175 & 0.552 & 0.263 & 0.168 \\
28.00 & 0.256 & 0.182 & 0.212 & 0.141 & 0.302 & 0.047 \\
28.40 & 0.301 & 0.043 & 0.249 & 0.008 & 0.341 & 0.008 \\
28.80 & 0.345 & 0.010 & 0.286 & 0.009 & 0.379 & 0.004 \\
\hline & & & & & &
\end{tabular}

a suitable pattern of triangular meshes to avoid self-crowding (see Saviane et al. 2000). The images with the artificial stars added were processed exactly in the same way as the original images (in particular photometry and calibration were done using the same parameters) and the photometry catalog was matched with the input artificial star list. It should be noticed that this procedure is approximated since it does not fully reproduce the spatial variations of the PSF. Still, it should account for the typical errors and completeness of the data. The results for photometric errors and completeness are presented in the form of completeness contours in the color-magnitude diagrams (see below) and in Table 1.

Figure 1 shows a color-image of our ground-based study (M02), highlighting some large-scale patterns like tails and holes in the distribution of the young blue stars. Figure 2 shows a true color image of SagDIG derived from our HST/ACS data. The spatial resolution of ACS allows us to resolve both the young and old component in SagDIG. It also confirms the large-scale patterns seen in ground-based images.

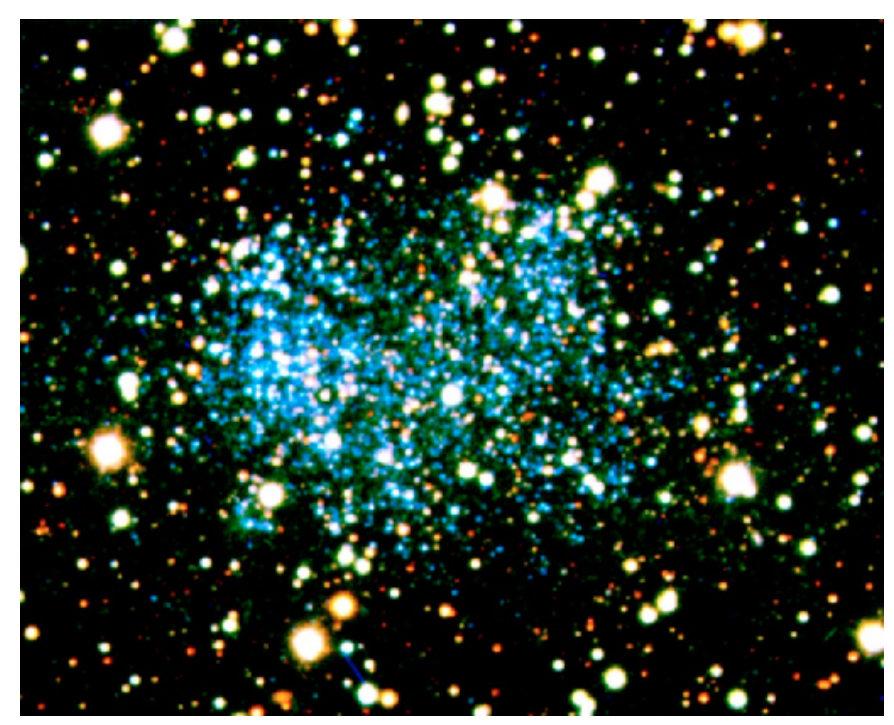

Fig. 1. A 3.1 $\times 2.4$ arcmin true-color image of SagDIG derived from our ground-based ESO/NTT BVI observations (1" resolution). North is to the top, East to the left. The low resolution of NTT data highlights large-scale structures such as tails of massive hot stars and regions almost devoid of young stars.

\section{Color-magnitude diagrams}

The ACS color-magnitude diagram of SagDIG reveals for the first time its history of star formation (Fig. 3). The high-resolution of HST/ACS allows overcoming the crowding difficulties, producing highly detailed CMDs where many distinctive features of the various stellar populations are seen for the first time. Figure 3 presents both the $m_{F 606 W},\left(m_{F 475 W}-\right.$ $\left.m_{F 606 W}\right)$ and the $m_{F 814 W},\left(m_{F 475 W}-m_{F 814 W}\right)$ diagrams, which complement each other in highlighting different evolutionary phases. The CMDs presented in this section are limited to stars with absolute SHARP values below 0.3.

Some foreground contamination is present in all diagrams. This is dramatic in the case of ground-based observations, yet it does not significantly affect the ACS data. Indeed, because of the distance of SagDIG, most of the galaxy stars are fainter than the main-sequence turnoff(s) of the thick disk stars, which are the main contributor to the foreground counts. In this respect, ACS really allows us to look through the Milky Way.

As an aid to the interpretation of our CMDs, we have simulated the color-magnitude diagram of the Galactic stars along the line of sight of SagDIG using the Besançon Galaxy Model (Robin et al. 2003; see also http:// bison.obs-besancon.fr/modele/). This is shown in the upper right panel of Fig. 3. The foreground stars belong to 3 main populations: (i) the Galactic disk, (ii) the thick disk, and (iii) the halo. Although the simulated diagram cannot be employed to quantitatively reproduce the observed Galactic contribution, because of the different pass-bands, the overall similarity of the simulated and observed diagrams is helpful to interpret the observed CMD features. The vertical sequence at $\left(m_{F 475 W}-m_{F 606 W}\right)=1.4$ is clearly produced by the superposition of the lower main sequences of Milky Way stars projected throughout the line of sight. The secondary peak located 


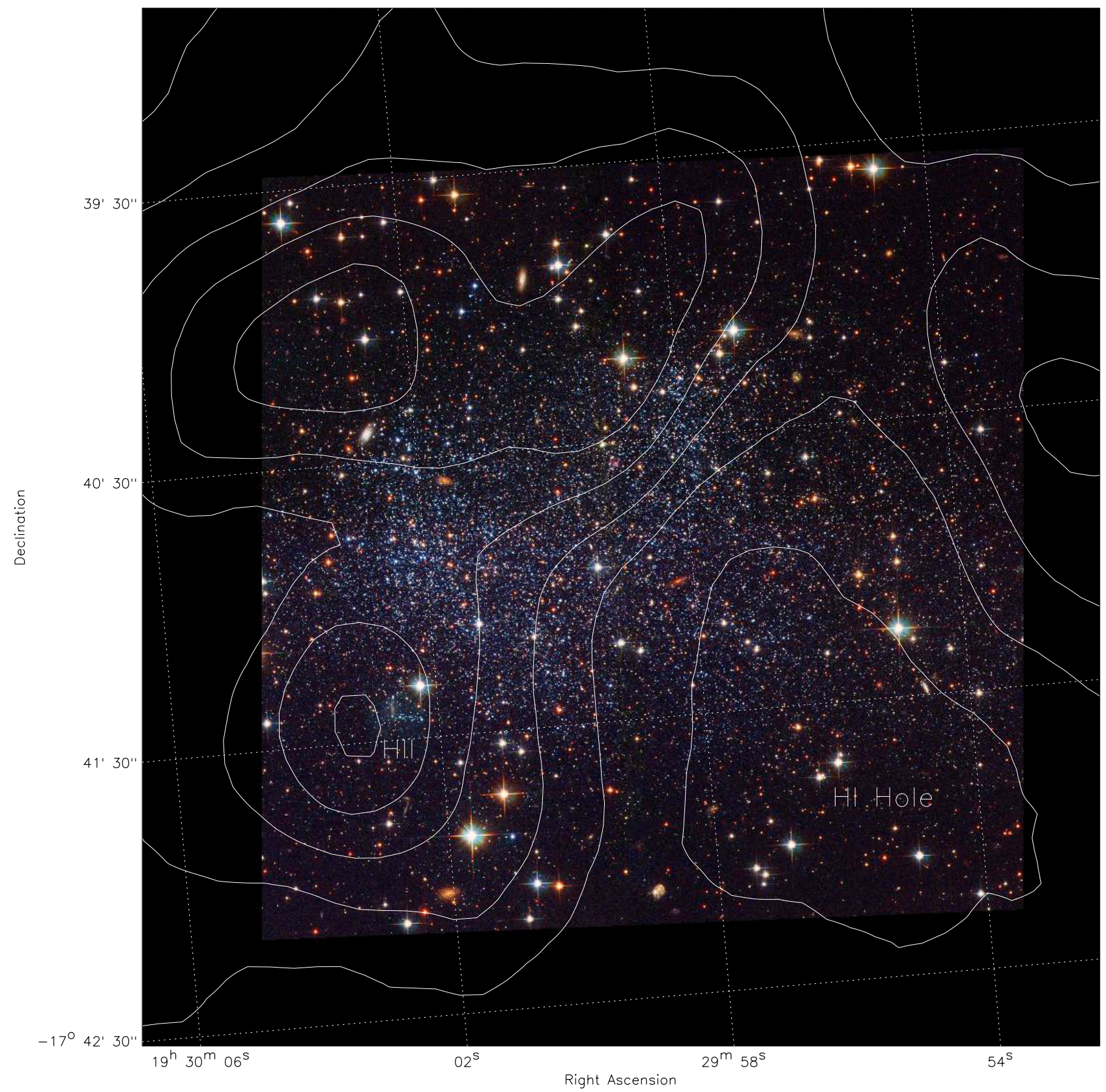

Fig. 2. True-color image of SagDIG derived from our ACS observations (see also http://heritage.stsci .edu/2004/31/index.html). A central, asymmetrical region of recent star formation, with faint extensions, is superimposed on a sheet of red giant stars belonging to the older stellar population of SagDIG. Superimposed are the H I column density contours from Young \& Lo (1997). Also plotted are the locations of the $\mathrm{HI}$ hole and $\mathrm{H}$ II region.

at $(B-V) \simeq 0.5$ corresponds to the overlapping of $\mathrm{G}$ field mainsequence turnoff stars along the line of sight. For example, stars at $m_{F 606 W} \simeq 20.0 \pm 0.2$ and $m_{F 475 W}-m_{F 606 W} \simeq 0.55$ would correspond to the turnoff area of Galactic stars located at $\sim 8 \mathrm{kpc}$ from us. These stars are quite important on their own, as they provide clues on the Galactic structure. A thorough analysis of the projected distribution of Milky Way stars will be possible at the completion of our program, when second-epoch data will enable us to analyze the proper-motion of all the observed populations.

Turning again our attention to SagDIG, we note that the red giant branch (RGB) is now well sampled from its tip at $m_{F 814 W} \approx 21.3$ down to $m_{F 814 W} \sim 26$, i.e. well below the red clump of core He-burning stars. This RGB is accompanied by a parallel and slightly bluer sequence of red supergiants (RSG), whose most luminous stars are confused with the Galactic foreground. The red super-giants are better seen in Fig. 4 (left panel), where we plot the CMD of stars in the inner region of SagDIG. This diagram is dominated by the young stellar population. On the other hand, the CMD of the outer regions (see right panel of Fig. 4) shows, as expected, a decreasing fraction of young stars.

One of the most noteworthy features of the ACS color-magnitude diagrams in Fig. 3 is the perfect separation 

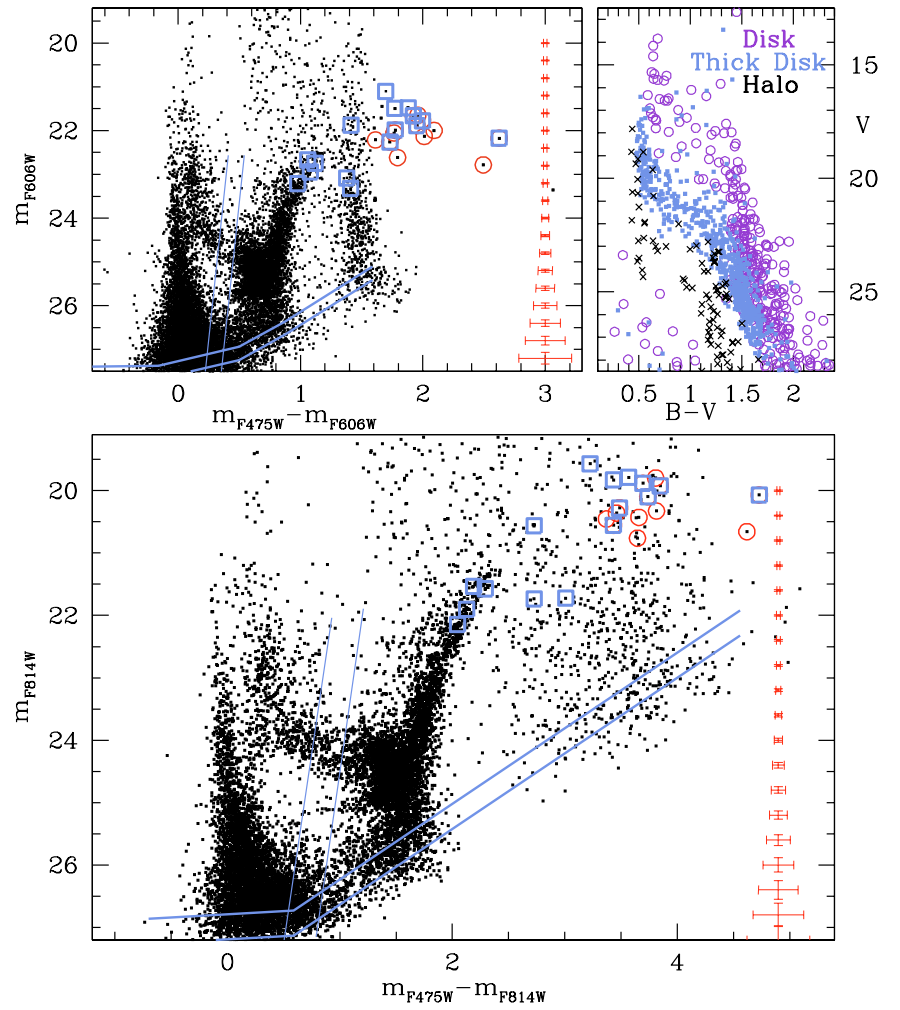

Fig. 3. HST/ACS color-magnitude diagrams of SagDIG. The upperleft panel shows the $m_{F 606 W},\left(m_{F 475 W}-m_{F 606 W}\right)$ diagram for stars with $|S H A R P|<0.3$. The thick lines represent the $80 \%$ and $50 \%$ completeness levels as derived from artificial stars experiments. Mean error bars from the same experiments are plotted as a function of $m_{F 606 W}$. The open symbols represent the carbon stars identified by Demers \& Battinelli (2002; open circles) and Cook (1987; open squares). The thin lines delimit the approximate location of the instability strip on the CMD. The upper-right panel shows a synthetic $V,(B-V)$ diagram in the direction of SagDIG, calculated using the WEB interface of the Besançon Galactic model (Robin et al. 2003). Different symbols refer to the contributions of the Galactic thin disk (open circles), thick disk (small squares) and halo (crosses). The lower panel shows the $m_{F 814 W},\left(m_{F 475 W}-m_{F 814 W}\right)$ diagram.

of the H-burning main sequence and the sequence of blue He-burning stars $(\mathrm{BHeB})$ in the blue-loops evolutionary phase. This is best seen in the $m_{F 814 W},\left(m_{F 475 W}-m_{F 814 W}\right)$ diagram of Fig. 3. Near $m_{F 814 W}=23$, the color difference between the two populations reaches $\left(m_{F 475 W}-m_{F 814 W}\right)=0.35$ mag. Besides the photometric precision of ACS, this result is obviously due to the wide color baseline chosen for our observations. At $m_{F 814 W} \simeq 25$, the MS shows a clear bifurcation. A small component departs from the main sequence and proceeds obliquely, crossing the blue loops at $m_{F 814 W} \sim 24.0$. This feature is indicative of a 200 Myr old burst of star formation (see Sect. 5). A similar feature is seen (although at a slightly lower resolution because of the narrower color baseline) in Sextans A (Dolphin et al. 2003).

We also notice a gap in luminosity between $m_{F 814 W}=20.6$ and 21.1 , in the distribution of blue super-giants centered at $\left(m_{F 475 W}-m_{F 814 W} \simeq 0.5\right)$. Although this hint is based on a statistically small sample of stars, we note that any quiescent starforming phase in the past 100 Myr would not be detectable along the MS, since successively younger stars would overlap, and therefore fill any empty region along the main sequence. On the contrary, evolved blue or red super-giants do not overlap in luminosity, therefore preserving any signature of a quiescent phase. Thus, the blue super-giant gap between $m_{F 814 \mathrm{~W}}=20.6$ and 21.1 (at $m_{F 475 W}-m_{F 814 W} \simeq 0.5$ ) might trace a short quiescent phase that we estimate (via isochrone fitting) took place some time between 30-60 Myr ago.

Another important feature in the CMD of SagDIG is the presence of an extended asymptotic giant branch (AGB) which traces a conspicuous intermediate age population. The AGB in SagDIG is partly masked by the foreground vertical sequence centered at $\left(m_{F 475 W}-m_{F 606 W}\right)=1.4$, and becomes really evident only when ACS photometric catalogs are crossidentified with the results of carbon star surveys. Figure 3 provides a cross-identification of our ACS data with the carbon star samples of Cook (1987; open squares) and Demers \& Battinelli (2002; open circles). This comparison confirms the composite nature of the SagDIG carbon stars sample. As suggested by Cook (1987), the C stars in SagDIG fall in two classes: (1) a luminous, intermediate-age population reaching $\sim 2 \mathrm{mag}$ in $I$ above the RGB tip; and (2) a bluer, low-luminosity component, overlapping the upper 1 mag of the RGB, probably belonging to an older population. We mention a third possibility for the origin of the carbon stars identified in the SagDIG field. Of the 3 stars that coincide with the vertical foreground sequence (upper-left panel in Fig. 3), the brightest one can be a luminous object on the extended AGB. However, the two fainter stars, quite separated from all other carbon stars, might belong to the Galactic foreground. These are possibly related to the population of faint high-latitude carbon stars found in the Sloan Digital Sky Survey (Downes et al. 2004). The possibility that these two stars are Galactic low-luminosity dwarf carbon stars will be better disentangled once our proper-motion analysis of SagDIG is completed.

The color-magnitude diagrams in Fig. 3 also show a prominent red clump (RC) of core He burning stars, with a complex, elongated shape. This structure (which will be quantitatively analyzed along with the star formation history of the galaxy in a separate paper - Held et al. 2005, in prep.) indicates that the bulk of SagDIG stars formed at intermediate ages ( $\gtrsim 1 \mathrm{Gyr})$, in a relatively extended star formation episode. The extension of the RC at higher luminosities, sometimes known as the "vertical clump" (VC), marks the evolution of younger stars ( 0.35-0.63 Gyr) in the "blue loop" core He-burning phase.

In addition to these components, we note a fainter extension at $m_{F 606 W}=25.70 \pm 0.05\left(m_{F 814 W} \sim 25.2\right)$ identified with a red horizontal branch (HB). Again, spatial selection helped us to pinpoint specific stellar populations. The presence of an old red HB is better seen in Fig. 4 (right panel), where stars located outside the regions of active star formation have been selected. Detecting the presence of a truly old population in SagDIG was one of the primary goals of this study and, indeed, this red HB implies that SagDIG hosts an old $(\gtrsim 10 \mathrm{Gyr})$ stellar population. Together, Figs. 3 and 4 may also suggest that a search for variable stars, in particular the old (>10 Gyr) RR Lyrae variables, might be worthwhile, even though the number of RR Lyrae stars we expect 


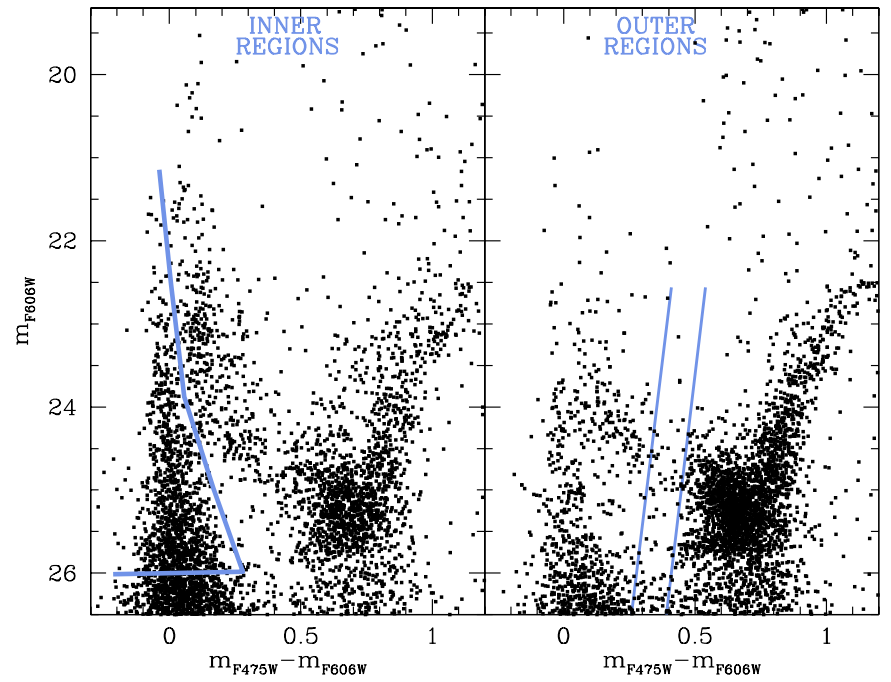

Fig. 4. Enlargements of the color-magnitude diagram illustrating the stellar population gradient in SagDIG. Left: the CMD of stars within the star forming regions, near the center of SagDIG. The curved line delimits the selection of MS stars used in calculating the star formation rate (see Sect. 5.2). Note the presence of the extended blue-loop feature and its red counterpart parallel to the RGB, indicating a significant population younger than $1 \mathrm{Gyr}$. This young population is less prominent in the right panel showing the $m_{F 606 W},\left(m_{F 475 W}-m_{F 606 W}\right)$ diagram for stars outside the star forming regions. The richness of the red clump and its complex structure are indicative of a predominant intermediate-age population formed over an extended epoch. The noticeable, although minor, extension to lower luminosity is reminiscent of the red horizontal branches seen in most dwarf spheroidal galaxies. The solid lines show the approximate location of the instability strip.

from Fig. 4 is not high. In these figures, the blue and red edges of the instability strip converted from the theoretical HR diagram (Chiosi et al. 1992) to the ACS/WFC system, have been over-plotted on the CMD. The "gaps" noticed along the HB $\left[\left(m_{F 475 W}-m_{F 606 W}\right)=0.3-0.4, m_{F 606 W} \simeq 25.8\right]$ as well as along the "vertical clump" $\left[\left(m_{F 475 W}-m_{F 606 W}\right)=0.35-0.45\right.$, $m_{F 606 W} \simeq 24.8$ ] show an almost perfect coincidence with the instability strip. In particular, the red edge appears to agree very well with a truncation in the red $\mathrm{HB}$ and blue-loop sequence. Indeed, short-period variable stars have been found in other dwarf irregular galaxies (e.g., Dolphin et al. 2002; Skillman et al. 2003; Baldacci et al. 2004). Unfortunately, a similar search cannot be conducted using our present observations because of their limited 3 hours baseline. Selection of candidate variable stars is likely to become possible once secondepoch observations are available.

\section{Reddening, metallicity, and distance \\ 4.1. Reddening}

The range of $E(B-V)$ values for SagDIG in the literature is not particularly large, spanning the interval between 0.06 (Lee \& Kim 2000) up to $E(B-V)=0.19$, derived in Saviane et al. (2002) from the Balmer decrement in an H II region. Nonetheless, this range may significantly affect our conclusions on the stellar metallicity in SagDIG. Momany et al. (2002) derived $E(B-V)=0.07$ from the colors of foreground

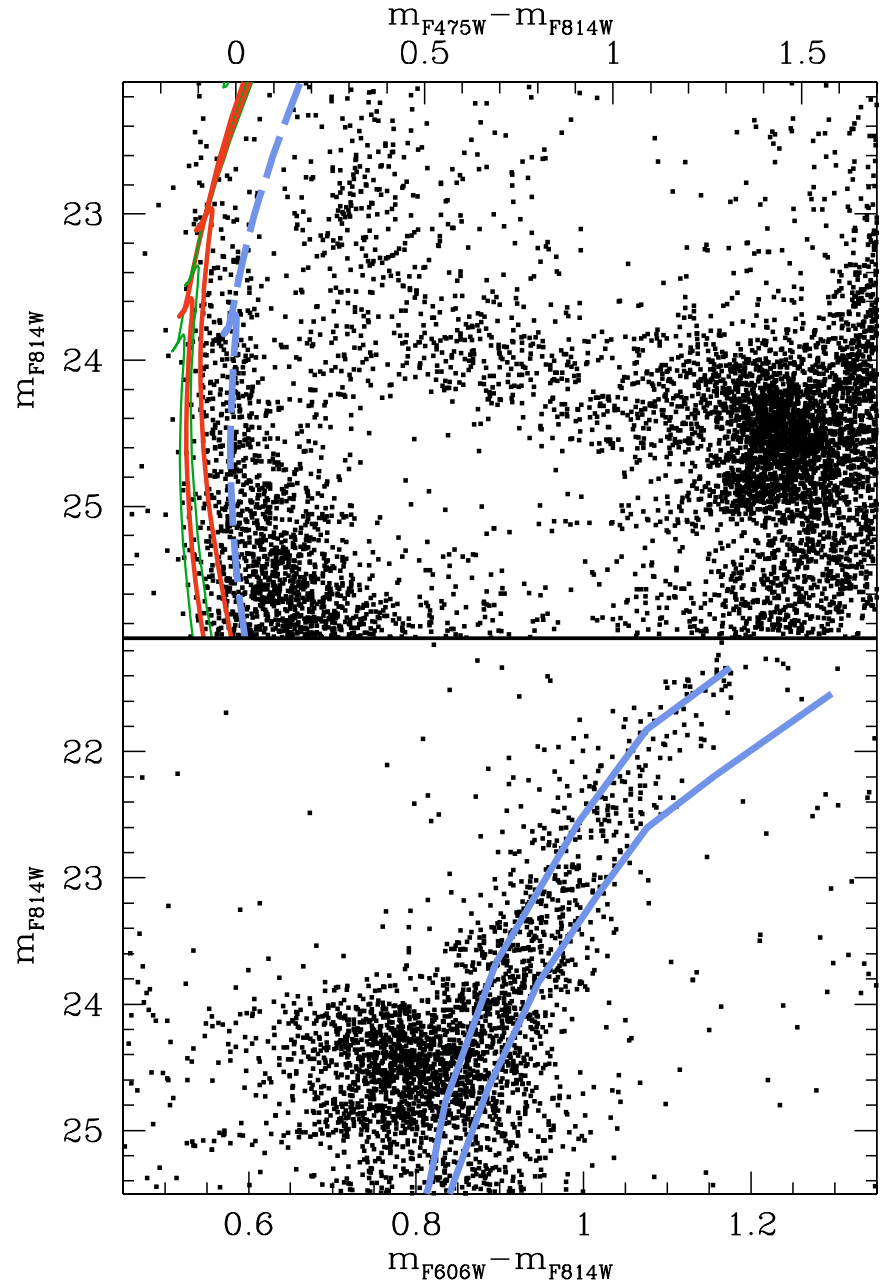

Fig. 5. The upper panel shows theoretical isochrones in the ACS-Vega system on the $m_{F 814 W},\left(m_{F 475 W}-m_{F 814 W}\right)$ diagram. Solid isochrones have been shifted using a $(m-M)_{0}=25.14$ and $E(B-V)=0.12$, whereas the long-dashed isochrone has been shifted using $E(B-V)=$ 0.20 . Solid isochrones, and from left to right, are with $Z=0.0001$, $0.0004,0.001$ and 0.004 (corresponding to $[\mathrm{Fe} / \mathrm{H}]=-2.36,-1.74$, -1.33 and -0.72). The long-dashed isochrone is with $Z=0.0004$. All isochrones refer to an age of $63 \mathrm{Myr}$. The lower panel shows the $m_{F 814 W},\left(m_{F 606 W}-m_{F 814 W}\right)$ color-magnitude diagram of SagDIG with superimposed the fiducial lines of the Galactic globular clusters NGC 6752 (right) and NGC 6341 (left), for which we used the reddening and distance moduli given in the Harris (1996) on-line catalog: http://physun.physics.mcmaster.ca/ harris/mwgc.dat, as updated in February 2003.

stars at the MS turnoff, while the Schlegel et al. (1998) extinction maps indicate a foreground reddening $E(B-V)=0.12$. These two values are likely to bracket the foreground reddening toward SagDIG.

In addition, some degree of internal reddening has been suggested by Momany et al. (2002) to be associated with the star-forming regions. Accordingly, a single value of reddening for all stellar populations in SagDIG may not be appropriate. A comparison of the ACS color-magnitude diagrams with de-reddened isochrones appears to confirm this earlier suggestion (Fig. 5, upper panel). In order to test the reddening value 
needed to fit the young main sequence, a set of 63 Myr old isochrones with different metallicities are over-plotted on the SagDIG color-magnitude diagram in the upper panel of Fig. 5. The isochrones provided by L. Girardi are from the Padua stellar evolution models, calculated in the same way as Girardi et al. (2002), using the ACS transformation curves by Sirianni et al. (2004). These isochrones assume $Y=0.230$. The conversion from $E(B-V)$ to the extinction in the ACS/WFC bands was taken from Bedin et al. (2004).

In the upper panel of Fig. 5 we over-plot 4 isochrones with $Z=0.0001,0.0004,0.001$ and 0.004 (respectively from left to right) shifted by $E(B-V)=0.12$. One sees that assuming this reddening value even the un-plausible hypothesis of metal-rich, 47 Tuc-like, isochrone is still bluer than the bulk of the SagDIG main sequence. On the contrary, a reasonable $Z=0.0004$ (as derived from the $[\mathrm{O} / \mathrm{H}]$ of the brightest $\mathrm{H}$ II region, Saviane et al. 2002) isochrone shifted by $E(B-V)=0.20$ shows an excellent fit to the SagDIG main sequence.

A similar comparison with isochrones cannot be employed for the RGB colors because of the well-known uncertainties in the color-metallicity calibration of theoretical isochrones for red giant stars (e.g., Westera et al. 2002). However, we can compare the data for SagDIG with the RGB fiducial lines of Galactic globular clusters to check the foreground reddening of RGB stars. The lower panel of Fig. 5 over-plots the RGB fiducial lines of two Galactic globular clusters, NGC 6341 and NGC 6752, onto the color-magnitude diagram of SagDIG. The clusters were observed with the same instrumental setup (GO 9453, P.I. Brown; see Brown et al. 2003) and re-calibrated by Bedin et al. (2004) with the same methods as used here for SagDIG. Both NGC $6341([\mathrm{Fe} / \mathrm{H}]=-2.3)$ and NGC 6752 $([\mathrm{Fe} / \mathrm{H}]=-1.54)$ have been corrected to the distance of SagDIG using the reddening and distance moduli given in the Harris (1996) catalog, and shifted using $E(B-V)=0.12$ and $(m-M)_{0}=25.14$ to match the SagDIG diagram. While this comparison does not provide itself an independent measurement of reddening, it gives no indication of any internal reddening outside of star-forming regions. Therefore in the case of RGB stars we adopt the Schlegel's et al. (1998) value as our estimate of the total reddening. In light of the results obtained in $\mathrm{M} 02$, we regard this reddening value as an upper limit and associate to it an uncertainty of \pm 0.05 that brackets the low reddening value adopted in M02.

\subsection{Metallicity of the red giant stars}

The mean stellar metallicity of SagDIG was estimated by analyzing a sample of red giant stars selected in regions outside the star-forming complexes. This was done using the Saviane et al. (2000) method, extended here to the $F 606 \mathrm{~W}$ and $F 814 \mathrm{~W}$ ACS pass-bands. The method is based on the construction of a family of hyperbolae in the plane $M_{F 814 \mathrm{~W}}$, $\left(m_{F 606 W}-m_{F 814 W}\right)_{0}$ suitable to fit the observed RGB fiducial lines of template globular clusters in a wide range of metallicities. The present implementation makes use of the empirical RGB fiducial lines of 3 Galactic globular cluster (NGC 104, NGC 6752, and NGC 6341) observed with ACS.

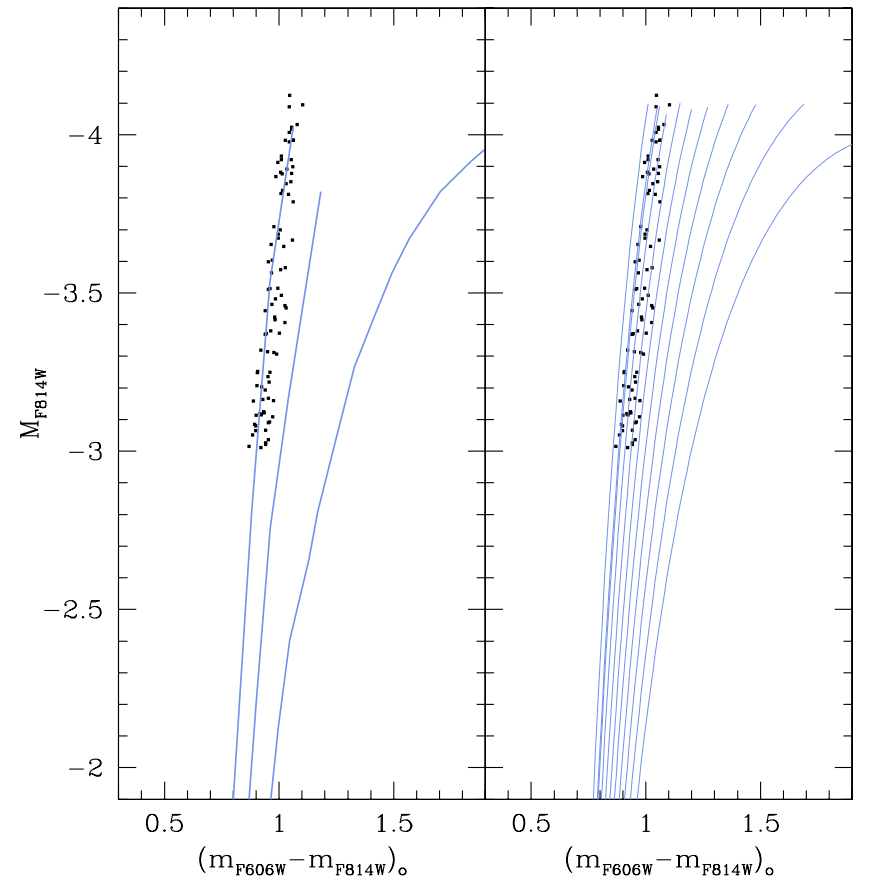

Fig. 6. The $2-\sigma$ selected sample of RGB stars in SagDIG, plotted on the $M_{F 814 W}$ vs. $\left(m_{F 606 W}-m_{F 814 W}\right)_{0}$ diagram. On the left panel, the data are shown along with RGB fiducial lines of the Galactic globular clusters NGC 6341, NGC 6752, NGC 104. A grid of analytic RGB templates are superposed to the data in the right panel (see text for details).

This set of globular clusters was originally observed by Brown et al. (2003) to constrain the metallicity of the M31 halo, and reanalyzed by Bedin et al. (2004). The adopted metallicity of the 3 clusters is $[\mathrm{Fe} / \mathrm{H}]=-0.76,-1.56$ and -2.28 , respectively, as given in the Harris (1996) catalog. The fiducial lines have been transformed into the $M_{F 814 W},\left(m_{F 606 W}-m_{F 814 W}\right)_{0}$ plane by adopting $(1)(m-M)_{V}=13.37,13.13$, and 14.64; and (2) $E(B-V)=0.04,0.04$, and 0.02 respectively.

The application of this method to SagDIG in the $M_{F 814 W}$, $\left(m_{F 606 W}-m_{F 814 W}\right)_{0}$ plane is illustrated in Fig. 6, where the absolute magnitude and reddening-corrected colors of individual stars in SagDIG are compared with the RGB ridge lines of the 3 Galactic clusters (left panel), and the grid of analytic RGB lines (right panel). The observed magnitude and colors have been transformed into the $M_{F 814 W},\left(m_{F 606 W}-m_{F 814 W}\right)_{0}$ plane by assuming $E(B-V)=0.12$ and $(m-M)_{0}=25.14$ (see below). The deepth of our ACS imaging allows a much better identification of the RGB with respect to ground-based observations. To prevent inclusion of young red super-giants contaminating the blue side of the RGB, we applied a $2 \sigma$ rejection in color about a fiducial line drawn by eye.

An interpolation over color provides a metallicity estimate for each star. The $[\mathrm{Fe} / \mathrm{H}]$ values of the selected RGB sample span from $[\mathrm{Fe} / \mathrm{H}]=-2.6$ to -0.8 . The resulting mean metallicity (for stars brighter than $M_{F 814 W}=-3.0$ ) is $\langle[\mathrm{Fe} / \mathrm{H}]\rangle=-2.21$ with an rms of $\sigma_{[\mathrm{Fe} / \mathrm{H}]}=0.18$. By repeating the calculation with a lower reddening, $E(B-V)=0.07$ (as in M02) we would obtain $\langle[\mathrm{Fe} / \mathrm{H}]\rangle=-1.89 \pm 0.17$. The value measured by Momany et al. (2002) was $\langle[\mathrm{Fe} / \mathrm{H}]\rangle=-2.10$. Given the uncertainties and the different pass-bands and calibrations, we 

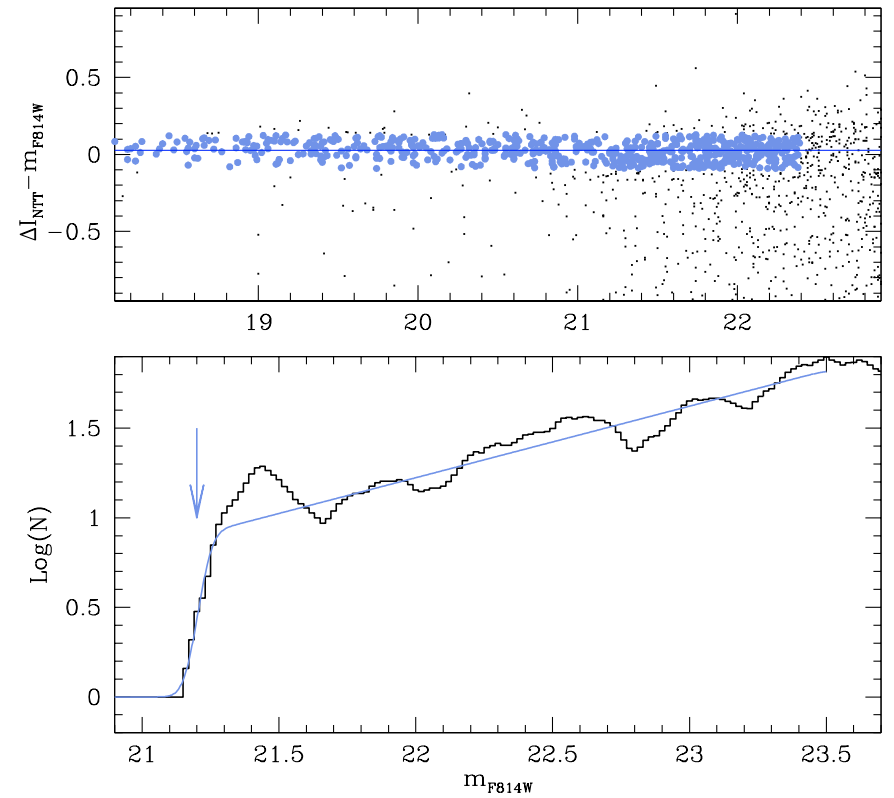

Fig. 7. The upper panel shows a comparison between the ACS $m_{F 814 W}$ data and $I_{\text {NTT }}$ photometry from Momany et al. (2002). Stars plotted with heavy symbols are a $3 \sigma$-clipped subsample yielding a mean shift of $-0.03 \pm 0.03 \mathrm{mag}$. The lower panel shows the luminosity function of SagDIG RGB sample selected outside the star-forming regions. The thin line represents the best fit with an error-convolved step function used to measure the RGB cutoff. An arrow marks the RGB tip at $m_{F 814 W}($ RGBT $)=21.23 \pm 0.05$.

regard these results as perfectly consistent. According to the new $[\mathrm{Fe} / \mathrm{H}]$ measurement, SagDIG is confirmed to be very metal-poor yet "normal" among dwarf irregular galaxies. We note that this metallicity estimate does not take into account the fact that the RGB stars in SagDIG are younger, on average, than the red giant stars in globular clusters. However, the effect is quite small for metal-poor stars (see Rizzi et al. 2003). We will implement this correction after discussing the SFH of SagDIG (E. V. Held et al. 2005, in prep.).

\subsection{Distance}

The excellent match between the RGB tip of SagDIG and that of GGCs obtained by assuming the distance derived by M02 suggests that the new ACS photometry is consistent with our previous distance estimate. Given the superior RGB obtained from ACS data, it appears worthwhile to verify the distance to SagDIG based on the magnitude of the RGB tip. Figure 7 shows the luminosity function of RGB stars in SagDIG together with our measurement of the tip. The RGB selection is the same as used for measuring the metallicity. The RGB tip was found at $m_{F 814 W}($ RGBT $)=21.23 \pm$ 0.05 by fitting an error-convolved step function as in M02. Since the color term for the filter $F 814 \mathrm{~W}$ is small in the color range of RGB stars (see Sirianni et al. 2004), we have been able to compare the new $F 814 \mathrm{~W}$ magnitudes with the ground-based $I$-band photometry of M02. The result is shown in Fig. 7, upper panel. The median difference is very small, $I_{\mathrm{NTT}}-m_{F 814 W}=+0.04 \pm 0.03$. By applying this correction, we obtain $I_{\text {tip }}(\mathrm{ACS})=21.27 \pm 0.05$. This is only $0.03 \mathrm{mag}$ brighter than the value measured in Momany et al. (2002), $I_{R G B T}=21.30$.

Assuming the new tip determination at $I=21.27 \pm 0.05$ and a reddening of $E(B-V)=0.12 \pm 0.05$ (i.e. $A_{I}=0.21$ comprising almost all reddening values reported in the literature) and adopting $M_{R G B T}^{I}=-4.04 \pm 0.05$ for metal-poor systems like SagDIG (see Da Costa \& Armandroff 1990; Lee et al. 1993; Bellazzini et al. 2001), the resulting distance modulus as derived from the calibration of the RGB tip is $(m-M)_{0}=25.10 \pm 0.11$. The distance error includes all uncertainties on the RGB tip determination and absolute calibration and reddening through standard error propagation. This basically confirms our previous M02 distance estimate, $(m-M)_{0}=25.14 \pm 0.18, \simeq 1.1 \mathrm{Mpc}$. The 0.04 smaller distance modulus obtained using the ACS data is to be attributed to a slightly brighter ( $0.03 \mathrm{mag})$ RGB tip determination, the assumed $M_{R G B T}^{I}$, and 0.05 higher $E(B-V)$. We note however, that calibrations of the absolute $I$ magnitude of the RGB tip are done in standard ground-based $I$ Cousins filter. Since the transformation from the ACS magnitudes to standard $I$ is quite uncertain, we retain our ground-based distance determination of SagDIG, and this is adopted for the rest of the paper.

\subsection{The horizontal branch}

Using this distance, Fig. 8 provides convincing evidence that the fainter extension of the RC identified in SagDIG is indeed a red horizontal branch. In order to estimate the magnitude level of the horizontal branch, we extract from the CMD stars in the color range $\left(m_{F 475 W}-m_{F 606 W}\right)=0.40-0.55$. The magnitude distribution of these (mostly core He-burning) stars is shown in Fig. 8 (upper-right panel) as a histogram with bin size 0.5 mag. A multi-bin luminosity function is plotted to reduce the effects of the choice of the bins. The red horizontal branch is clearly detected at $m_{F 606 W}=25.70 \pm 0.05$. This is confirmed when we over-plot, in the upper-left panel, a Zero-Age HB (ZAHB) model of $[\mathrm{Fe} / \mathrm{H}] \simeq-2.1$ from Pietrinferni et al. (2004), having assumed $E(B-V)=0.12$ and $(m-M)_{0}=25.14$, that perfectly fit the estimated level of HB in SagDIG.

Further evidence for the correct identification of the $\mathrm{HB}$ is given in the lower panels of Fig. 8, where we compare the HB morphology in SagDIG with (i) the HB of an old, metalpoor Galactic globular cluster, namely NGC 2419, and (ii) the ACS observations of variable stars in M 31 (Brown et al. 2004).

NGC 2419 is one of the few clusters matching the SagDIG metallicity and observed in the same ACS filters (GO 9666, P.I. Gilliland). Also plotted is a sketch of the instability strip. For the HB stars in NGC 2419 bluer than $m_{F 475 W}-m_{F 814 W}=$ 0.5 we only plot a fiducial line because of the overlap with the young MS stars in SagDIG. This comparison shows the consistency of the location the HB level in SagDIG with that observed in the oldest globular clusters. On the other hand, it lends support to the adopted distance modulus and reddening.

In addition, in Fig. 8 our data are compared with ACS observations of RR Lyrae stars in M 31 (Brown et al. 2004). In this case, we have arbitrarily adjusted the M31 data to match 


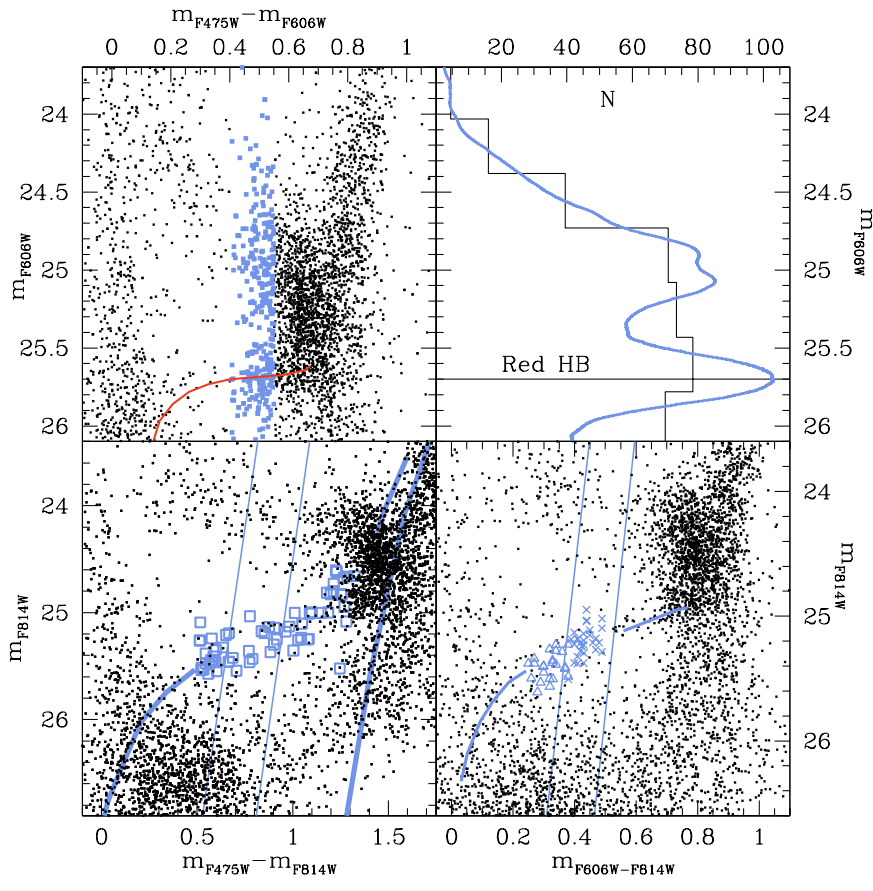

Fig. 8. Enlargements of the CMDs illustrating the detection of an old horizontal branch in SagDIG. The upper-left panel highlights the color interval used for measuring the magnitude distribution of He-burning stars, along with a $[\mathrm{Fe} / \mathrm{H}] \simeq-2.1 \mathrm{ZAHB}$ model (assuming $Y=0.245$ ) from Pietrinferni et al. (2004). In the upper-right panel we plot the histogram and a multi-bin, smoothed luminosity function of stars selected in upper-left panel. A line at $m_{F 606 \mathrm{~W}} \sim 26.70$ marks the level of the old red-HB. In the lower-left panel, we have superimposed on the SagDIG data the fiducial loci for the globular cluster NGC 2419 (Momany et al. 2005, in prep.). These data were fully calibrated in the same way as for the SagDIG stars. The HB stars in NGC 2419 in the color interval $0.4<m_{F 475 W}-m_{F 814 W}<1.4$ are shown as open squares. The Brown et al. (2004) data for RR Lyrae variable stars in M 31 are shown in the lower-Right panel (crosses: RRab; triangles: $\mathrm{RRc}$ ). Also plotted is the fiducial line for static HB stars in M31. The Brown's et al. data have been arbitrarily shifted to match the red-HB in SagDIG.

the red-HB of SagDIG. This comparison suggests where one should search for RR Lyrae variable stars.

\section{The recent star formation}

\subsection{Blue loop stars: age and metallicity}

Theoretical isochrones can now be used to characterize the ages of the MS and blue HeB stars. Since the metallicity of SagDIG is known to be in the metal-poor regime, we employed isochrones with $Z=0.0001$ and $0.0004([\mathrm{Fe} / \mathrm{H}]=$ -2.31 and -1.73 , respectively) for our comparison with observational data. Very metal-poor isochrones $(Z=0.0001)$ with ages $31.5,100,200$, and $630 \mathrm{Myr}$ are over-plotted on the $m_{F 814 W},\left(m_{F 475 W}-m_{F 814 W}\right)$ CMD of SagDIG in the upper panel of Fig. 9, while isochrones with $Z=0.0004$ and ages of 31.5, 89, 200 and $630 \mathrm{Myr}$ are shown in the lower panel. The MS and blue loops are quite well reproduced by both sets of isochrones from the brightest stars $\left(m_{F 814 W}=20.5\right)$ down to the "vertical clump". However, the excursion of the blue loops

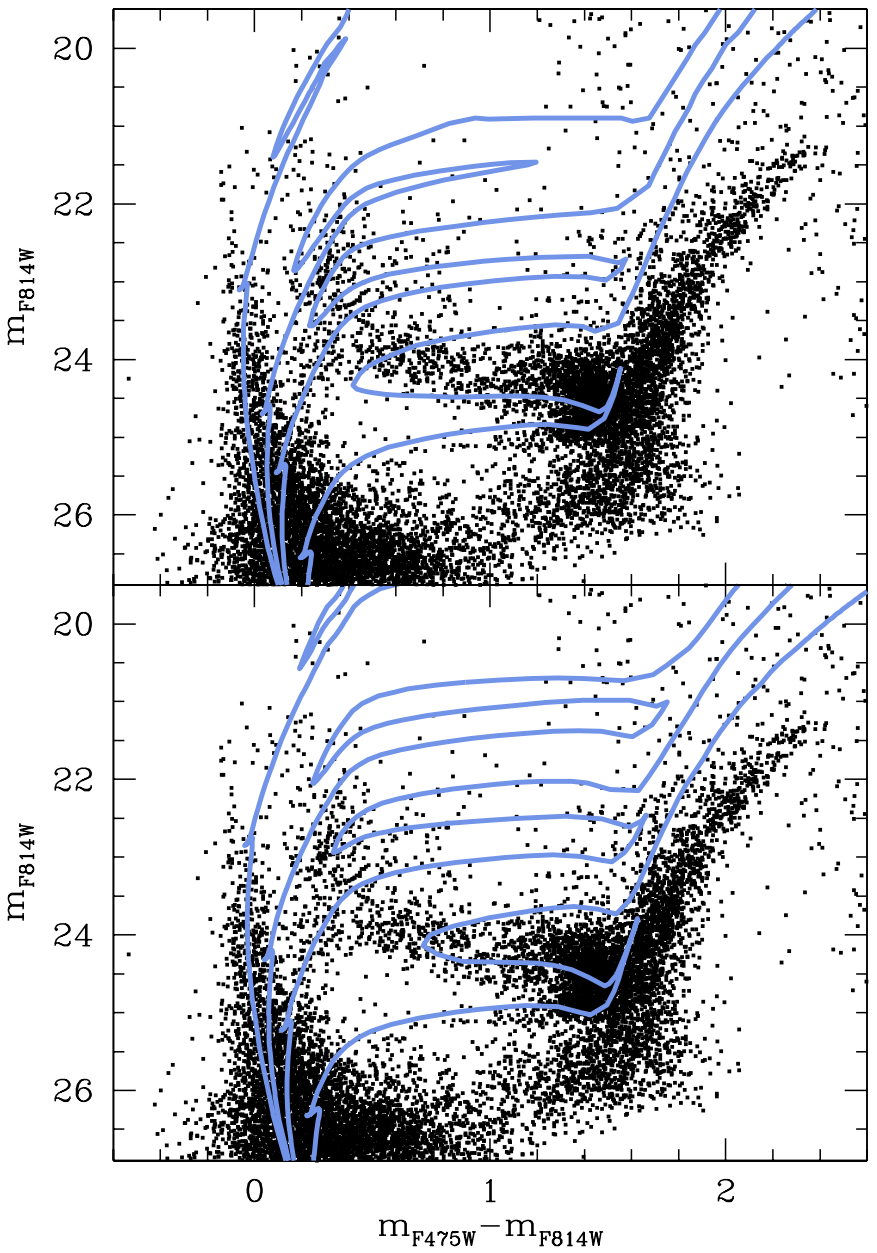

Fig. 9. Model isochrones from Girardi et al. superimposed on the $m_{F 814 W},\left(m_{F 475 W}-m_{F 814 W}\right)$ diagram of SagDIG. Upper panel: $Z=0.0001$ isochrones with ages of 31.5, 100, 200, 630 Myr. Lower panel: $Z=0.0004$ isochrones with ages $31.5,89,200,630 \mathrm{Myr}$.

in the isochrones, which is strongly correlated to metallicity, favors a less extreme metal-poor composition $(Z=0.0004$, or $[\mathrm{Fe} / \mathrm{H}] \sim-1.7$ ) for the $100-600$ Myr old stars in SagDIG. The details of stellar evolution of $\mathrm{HeB}$ intermediate-mass stars depend on many uncertain physical parameters (such as the amount of convective overshooting, stellar rotation, and mass loss), so evolutionary tracks cannot be safely used to measure the metallicity of young HeB stars. However, the Padua model isochrones are known to provide a good fit to systems with known age. Therefore, assuming that metallicity is the main driver of the morphology of the blue loops (but having in mind all the systematic uncertainties on the model physics), this comparison would suggest a modest chemical enrichment $(\sim 0.4$ dex $)$ in stars since the main star formation episode from which most of the RGB stars originated.

\subsection{Star formation rate from main sequence stars}

While a full analysis of the star formation history of SagDIG based on synthetic color-magnitude diagrams will be presented elsewhere, we give here a first account of the mean star formation rate (SFR) in the last 0.6 Gyr. The SFR was 


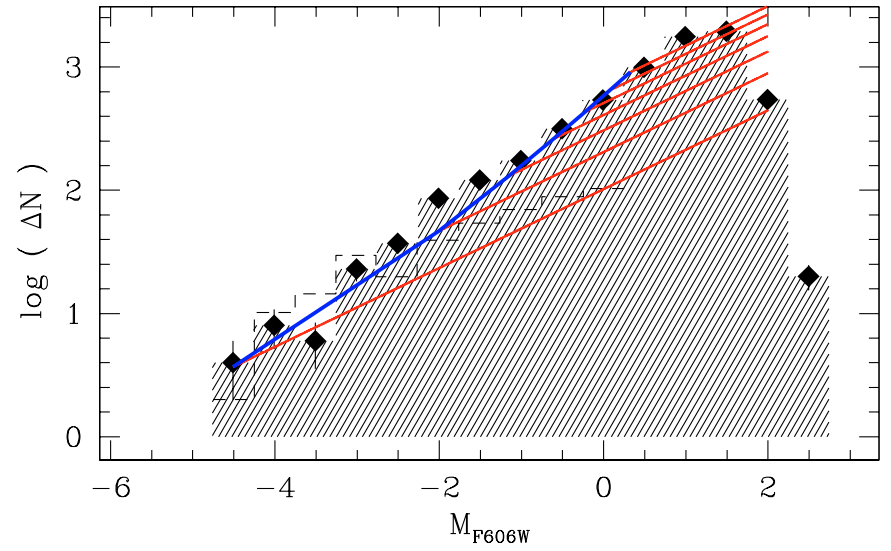

Fig. 10. The $M_{F 606 W}$ luminosity distribution of MS stars in SagDIG (shaded histogram). The thick solid line is our best-fitting model, which can be regarded as the superposition of luminosity functions of many SSPs (thin solid lines). The LF of the young LMC cluster NGC 2004 is shown as a dashed histogram.

computed as in Saviane et al. (2004; hereafter SHR04), using the equation:

$\log \dot{n}=\left(\alpha_{\mathrm{CSP}}-\alpha_{\mathrm{SSP}}\right) \Delta M_{V}-\log \Delta t$

where $\alpha_{\text {SSP }}$ and $\alpha_{\text {CSP }}$ are the slopes of the luminosity function (LF) of a simple (isochronic) and a composite stellar population, respectively. $\Delta M_{V}$ and $\Delta t$ are the luminosity and age interval over which the mean SFR is calculated. All the parameters in the equation are measured on the $V$ main sequence LF, except for $\Delta t$ which is obtained from the theoretical isochrones.

The main-sequence LF of SagDIG was obtained by selecting MS stars as indicated in Fig. 4, and then constructing the $M_{V}$ histogram with a bin size of $0.5 \mathrm{mag}$ (Fig. 10) ${ }^{1}$. The absolute magnitudes were computed as usual from the relation $M_{V}=V-(m-M)_{0}-R_{V} E(B-V)$, adopting a corrected distance modulus $(m-M)_{0}=25.14 \pm 0.18$, a reddening $E(B-V)=0.19 \pm 0.04$ (appropriate for the MS stars, see Sect. 4.1), and a ratio $A_{V} / E(B-V)=R_{V}=3.1$. The mean slope of the LF of SagDIG, $\alpha_{\mathrm{CSP}}=0.486 \pm 0.022$, was calculated by fitting a straight line to the LF for $V \leq 26$. This ensures that stars on the sub-giant branch do not contaminate the MS sample. Given this limit, the useful LF interval is $\Delta M_{V}=5 \mathrm{mag}$. In order to calculate the corresponding age interval, we used the interpolation of the MS termination against age given in SHR04. The luminosity range of the LF corresponds to age limits from 676.7 Myr ago to 20.4 Myr ago, implying $\Delta t=656.2$ Myr. A slope $\alpha_{\text {SSP }}=0.32$ was adopted for simple stellar populations, as in SHR04.

According to Eq. (1), we find a SFR $\dot{n}=1.032 \times$

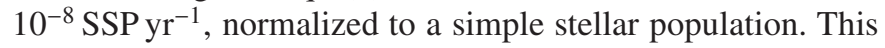
means that in the quoted time interval $(\sim 0.6 \mathrm{Gyr}), 6.8 \mathrm{SSPs}$

\footnotetext{
${ }^{1}$ We converted the $m_{F 606 \mathrm{~W}}$ photometry of MS stars into $V$ magnitude by computing a mean difference $\left\langle V_{\mathrm{NTT}}-m_{F 606 \mathrm{~W}}\right\rangle=+0.05 \mathrm{mag}$ (Momany et al. 2002). This procedure is acceptable for MS stars since we are considering a narrow color range around $(B-V) \simeq 0$, while in general the mismatch with the Johnson $V$ band is too large to allow a meaningful transformation. For example, for the Galactic foreground sequence at $\left(m_{F 475 W}-m_{F 606 W}\right) \simeq 1.40,\left\langle V_{\mathrm{NTT}}-m_{F 606 W}\right\rangle=-0.50$.
}

were created. Finally, based on the zero-point difference between the LF of our template (the young LMC cluster NGC 2004) and a single SSP, we find that one SSP here contains 1.32 clusters like NGC 2004 , or $2.65 \times 10^{4} M_{\odot}$ (see SHR04 for further details on the method). Therefore the mean starformation rate in the quoted interval is $2.65 \times 10^{4} \times \dot{n}=$ $2.73 \times 10^{-4} M_{\odot} \mathrm{yr}^{-1}$.

Karachentsev et al. (1999; KAM99) computed the SFR of SagDIG through a simulation of the observed color-magnitude diagram, and found $S F R=13 \pm 5 \times$ $10^{-4} M_{\odot} \mathrm{yr}^{-1}, 6.6 \pm 0.8 \times 10^{-4} M_{\odot} \mathrm{yr}^{-1}$, and $1.3 \pm 0.1 \times$ $10^{-4} M_{\odot} \mathrm{yr}^{-1}$ in the age ranges $0-0.05 \mathrm{Gyr}, 0.05-0.2 \mathrm{Gyr}$, and $0.2-15$ Gyr, respectively. In order to compare these values with our rate, we computed the time-weighted SFR of KAM99 within our time interval (0-0.677 Gyr), which is $3.3 \times 10^{-4} M_{\odot} \mathrm{yr}^{-1}$, or $\sim 20 \%$ larger than the SFR computed above. Now KAM99 point out that the age range $0.2-1$ Gyr is poorly sampled by their CMD, so our value is perfectly compatible with their SFRs. A further explanation for the reported difference is that KAM99 computed their SFR over an area which is larger than our ACS field.

If we restrict our SFR computation to the 0-0.24 Gyr interval (i.e. cutting the $\mathrm{LF}$ at $V=24.5$ ), we obtain a rate similar to the previous one $\left(2.6 \times 10^{-4} M_{\odot} \mathrm{yr}^{-1}\right)$, while KAM99 find $6.6 \times 10^{-4} M_{\odot} \mathrm{yr}^{-1}$. One possibility is that the larger SFR of KAM99 is due to an incorrect evaluation of the MS/blueloop ratio. This would not be surprising, given the large errors affecting ground-based photometry, that do not allow a clear separation between the two sequences (as it is the case in the ACS data). We also note that KAM99, using the $\mathrm{H} \alpha$ flux measured by Strobel et al. (1991), i.e. $28.1 \times 10^{-15} \mathrm{erg} \mathrm{cm}^{-2} \mathrm{~s}^{-1}$, and applying the method of Aparicio et al. (2000), find a current $S F R=1.5 \times 10^{-4} M_{\odot} \mathrm{yr}^{-1}$, much closer to that which we find here. In summary, our method predicts that the recent SFR remained almost constant during the last few hundreds of Myrs. The SFH of SagDIG will be further considered with CMD simulations of the present ACS data in a future paper.

\section{The spatial distribution of gas and stars}

The interplay between stars and the surrounding interstellar matter (ISM) in the form of neutral, ionized, and molecular gas, is a key aspect in understanding what triggers star formation and drives the wide range of properties observed in dwarf galaxies. To investigate this connection, we analyze here the distributions of stars of different ages in SagDIG from our HST/ACS observations and compare them with the distribution of the ISM.

\subsection{The distribution of the gas}

We begin our discussion by summarizing here the properties of the ISM in SagDIG. The observations of H I in dwarf galaxies often decompose the neutral $\mathrm{H}$ into two components (see, e.g., Young et al. 1997, and references therein). In the particular case of SagDIG, a warm (broader and more turbulent) H I component having a velocity dispersion $\sigma=10 \mathrm{~km} \mathrm{~s}^{-1}$ was observed, along with a cold (narrower and more quiescent) phase 
with $\sigma=5 \mathrm{~km} \mathrm{~s}^{-1}$ (Young \& Lo 1997). The broad component is distributed all over the galaxy, whereas the narrower component is concentrated in few H I clumps of about $8 \times 10^{5} M_{\odot}$ (6 clumps are listed in Table 1 of Young \& Lo 1997). A prominent H I clump (with a column density $1.1 \times 10^{21} \mathrm{~cm}^{-2}$ ) is nearly coincident with the biggest, and probably only, H II region in SagDIG. This H II region is $100 \mathrm{pc}$ across, and $100 \mathrm{pc}$ away from the H I clump center (Strobel et al. 1991). These H I clumps form an almost complete asymmetric ring (or shell) structure surrounding a region of lower H I column density, hereafter referred to as the "H I hole". The roundness of the outer H I distribution led Young \& Lo to suggest an almost faceon orientation of SagDIG. The diameter of the ring is nearly $4 \operatorname{arcmin}(1.3 \mathrm{kpc})$, while the largest extent of the $\mathrm{HI}$ at a column density of $5 \times 10^{18} \mathrm{~cm}^{-2}$ is $3.3 \times 3.0 \mathrm{kpc}$.

Young \& Lo (1997) found very little systematic rotation in SagDIG, i.e. any rotation velocity is of the order of, or smaller than, the intrinsic velocity dispersion. However, some degree of symmetry in the velocity field led the authors to suggest the presence of some large-scale organized motion.

Young \& Lo (1997) also suggested that the cold dense atomic phase tells us where conditions are right for the formation of molecular gas and stars. The sizes of the H I cold components imply virial masses which are a few times larger than the H I mass alone; the difference may be caused by molecular gas. Thus, the cold H I component may trace the raw ingredient necessary for any star formation activity. Indeed, it is found in clumps of about the same size as the star formation regions. In dwarf irregulars, star formation typically occurs in regions of 100-200 pc in size, with each episode lasting for about $100 \mathrm{Myr}$ and then moving around (see, e.g., Dohm-Palmer et al. 1997, 1998, for the case of Sextans A).

\subsection{Spatial distribution of young and old stellar populations}

The unique photometric deepness and precision of our ACS data allowed us not only to disentangle stars in different evolutionary phases (e.g., main-sequence stars against blue supergiants) but also to assign reliable age estimates to them from a comparison with the Padua isochrones on the WFC/ACS system (Girardi 2004, priv. comm.). Figure 11 shows the distribution of stars in different age intervals superimposed on the H I contour map of Young \& Lo (1997), kindly provided by the authors. Astrometric calibration of the radio map and our optical $F 606 \mathrm{~W}$ reference image was performed independently and carefully checked. In all panels, the HST/ACS footprint is superposed on the H I map so as to mark the boundaries of our field of view. We note that, in order to prevent distribution biases caused by the spatial variations in the PSF of WFC/ACS, no selection based on image shape parameters was applied to the photometry catalogs when plotting these spatial distributions. For instance, we found that spurious distributions may originate from selection on the $|S H A R P|$ parameter due to the spatially varying image quality.

The location of the youngest stars in SagDIG, i.e. the mainsequence stars with age less than $70 \mathrm{Myr}\left(m_{F 606 W} \leq 24.0\right)$, is plotted in Panel A of Fig. 11. The MS and BSG stars were selected on the left and right side, respectively, of the separation line outlined in Fig. 4. The most evident feature is the starforming region located in between the H I clumps $i$, ii and iii on the eastern part of SagDIG. The angular size of this complex is $\sim 30^{\prime \prime}$, or $155 \mathrm{pc}$, in diameter. Very recent star formation is also seen in the central part of the optical galaxy, on the rim of the H I "hole", in between the H I clumps $i v$ and $v$. A very small complex, or a star cluster, with only a few very massive stars is seen close to the SE hydrogen clump $(i)$. This cluster is of particular interest being associated with the only $\mathrm{H} \mathrm{II} \mathrm{re-}$ gion in SagDIG. Panel B plots the distribution of MS stars having age between 70 and $160 \mathrm{Myr}\left(24.0 \leq m_{F 606 W} \leq 25.0\right)$. The stellar distribution starts to be more widespread, with a tail extending out to the H I clump $(v)$ on the western side. The blue-loop super-giants in the same age range 70-160 Myr $\left(m_{F 606 W} \leq 23.0,0 \leq m_{F 475 W}-m_{F 606 W} \leq 0.2\right)$, shown in Panel C, are consistent with this pattern, although their number is very small. Panel D of Fig. 11 summarizes the distribution of the most recent star formation in SagDIG (age $<160 \mathrm{Myr}$ ), showing its nearly circular symmetry, with a prominent eastern starforming complex and a peculiar tail of massive stars reaching the westernmost hydrogen cloud.

The distribution of older MS stars $\left(25.0 \leq m_{F 606 W} \leq 26.5\right)$ and blue super-giants $\left(22.5 \leq m_{F 606 W} \leq 24.8, m_{F 475 W}-\right.$ $m_{F 606 W}<0.4$; age between 160 and $700 \mathrm{Myr}$ ) in shown in Panels E and F. The eastern star-forming region, which is main site of very recent star formation, is also present for stars older than 160 Myr. In this age range, however, a complex on the western side of H I cloud $i v$ becomes also prominent. The latter appears to have been the most active star-forming region more than a few hundred million years ago.

Figure 11 gives us a clear picture of the relationship between the HI and the recent star formation $(\leq 700 \mathrm{Myr})$ in SagDIG. It confirms the earlier finding by Young \& Lo (1997) that recent star formation sites are always near (although not coincident) with the H I column density peaks. This is best seen for the eastern and western SF complexes, as well as the small association coincident with the $\mathrm{H}$ II region.

The spatial distribution of relatively old stars, including main-sequence stars older than $0.7 \mathrm{Gyr}$, He-burning stars on the red clump and the red-HB, and RGB stars, is shown in Fig. 12. Panel A shows the distribution of bright RC stars $\left(24.8 \leq m_{F 606 W} \leq 25.4,0.5 \leq m_{F 475 W}-m_{F 606 W} \leq 0.7\right)$. A comparison with isochrones indicates for these stars an age interval between $300 \mathrm{Myr}$ and $2.0 \mathrm{Gyr}$. The distribution of the old red-HB stars $\left(25.6 \leq m_{F 606 W} \leq 25.8,0.4 \leq m_{F 475 W}-m_{F 606 W} \leq\right.$ 0.6) is shown in Panel B. These stars are most likely older than 9-10 Gyr. A similar plot is done for RGB stars brighter than $m_{F 606 W}=24.50$ in Panel C.

For the stars younger than $1 \mathrm{Gyr}$, a correlation with the distribution of the gas and the star-forming regions can still be noticed. The red clump stars, for instance, appear to follow the the rims of H I clumps on the eastern side. Yet, there is no particular hint of a H I hole imprint. This trend is not present anymore in RGB stars (older than $1 \mathrm{Gyr}$ ) which show some central concentration. For the genuinely old (red-HB) population the 


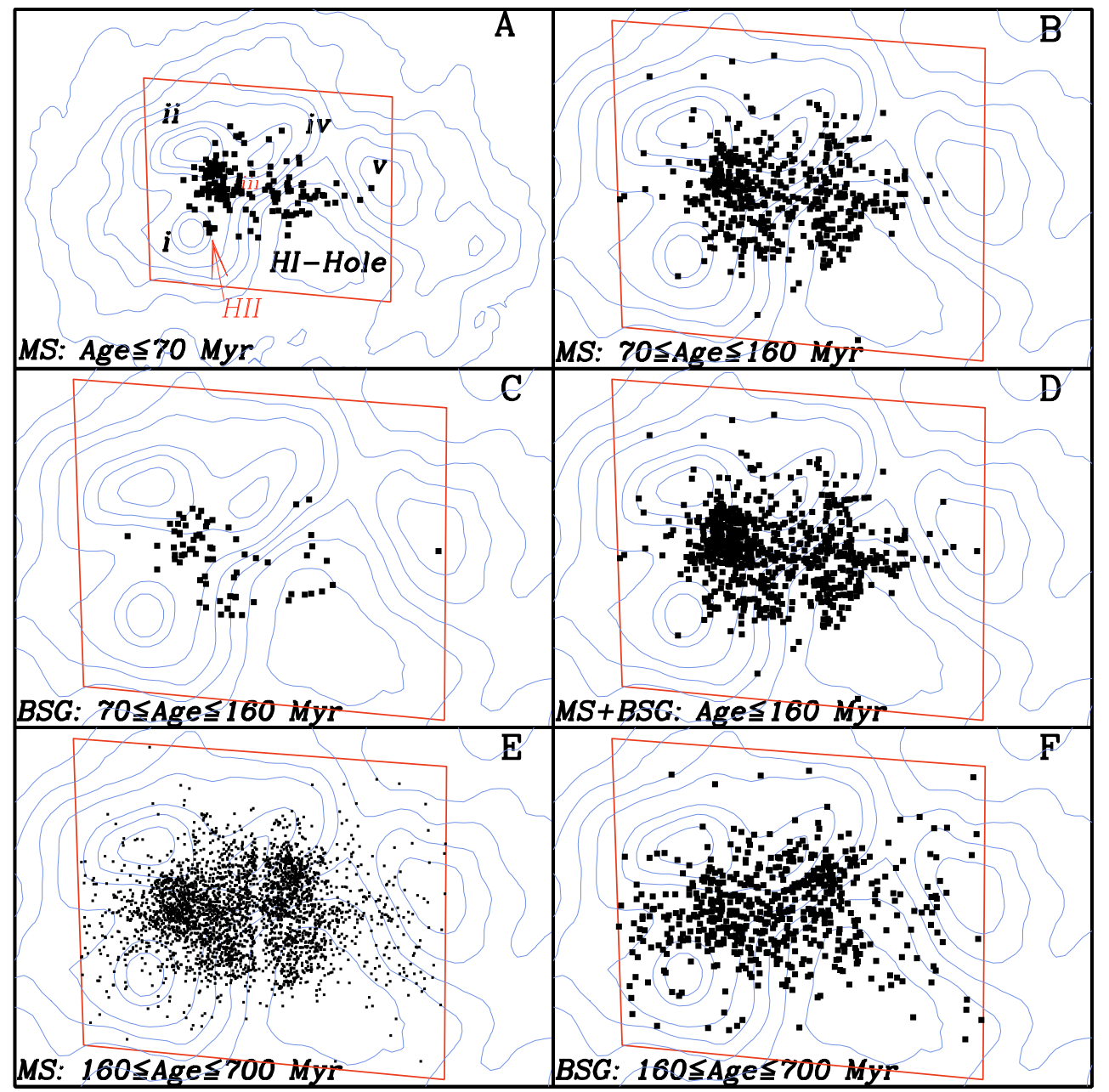

Fig. 11. Spatial distribution of MS and BSG stars of different ages superimposed on the H I contours from Young \& Lo (1997). North is to the top and East to the left. Also plotted is the HST/ACS footprint. Panel A) MS stars with $m_{F 606 W} \leq 24.0$ and age less than 70 Myr. Panel B) MS stars with $24.0 \leq m_{F 606 W} \leq 25.0$ and age between 70 and 160 Myr. Panel C) BSG stars with $m_{F 606 W} \leq 23.0$, age 70-160 Myr. Panel D) all MS and BSG stars younger than 160 Myr. Panel E) MS stars with $25.0 \leq m_{F 606 W} \leq 26.5$, age interval 160-700 Myr. Panel F) BSG stars $22.5 \leq m_{F 606 W} \leq 24.8$, age between 160 and $700 \mathrm{Myr}$.

statistics is really poor - all we can say is that their distribution is rather uniform and extended.

It is interesting to compare these distributions with the location of MS stars older than 0.7 Gyr $\left(26.5<m_{F 606 W}<27.50\right.$, Panel D). The fainter limit roughly corresponds to our $50 \%$ incompleteness level, or an age of about 2.0 Gyr. The H I hole region is smoothly populated, and the star distribution is - already in this age interval - fairly uniform with an overall central concentration, and extended. There are no detectable voids in the distribution of stars. However, the distribution is clearly inhomogeneous, still showing the presence of the north-western clump, and a generally higher surface density of stars on the eastern region. This implies that the sites of recent star formation discussed for the younger stars, in particular the northwestern site, are quite old being already in place 1-2 Gyr ago.

\subsection{The HI hole}

If the large H I hole in SagDIG is a structure that has formed over time from an initially more uniform distribution we can attempt to address the question of how it formed using constraints from our deep ACS imaging. Before we continue however we should stress that the assumption that the hole formed from a uniform distribution of atomic gas may be unfounded. This point is discussed at the end of this section.

A popular scenario for the formation of $\mathrm{HI}$ holes is as the result of the energy input to the ISM from massive stars in the form of stellar winds and more importantly supernovae. Several galaxies have been observed with H I holes, and in some cases these holes are observed to be expanding, although this is generally observed only for small holes (refer to HoII, M33, LMC, SMC, M 31, Sextans A). Opinion is divided, even for a given galaxy, as to whether the supernova-driven wind hypothesis is viable or not (see for example Rhode et al. (1999) and Stewart et al. (2000)).

If the H I hole in SagDIG formed from the combined energetic input of supernova explosions we might expect to detect the light from those stars which formed along with those that drove the expansion but that have not exploded as supernovae. We would expect these stars to be found in a cluster or clusters 


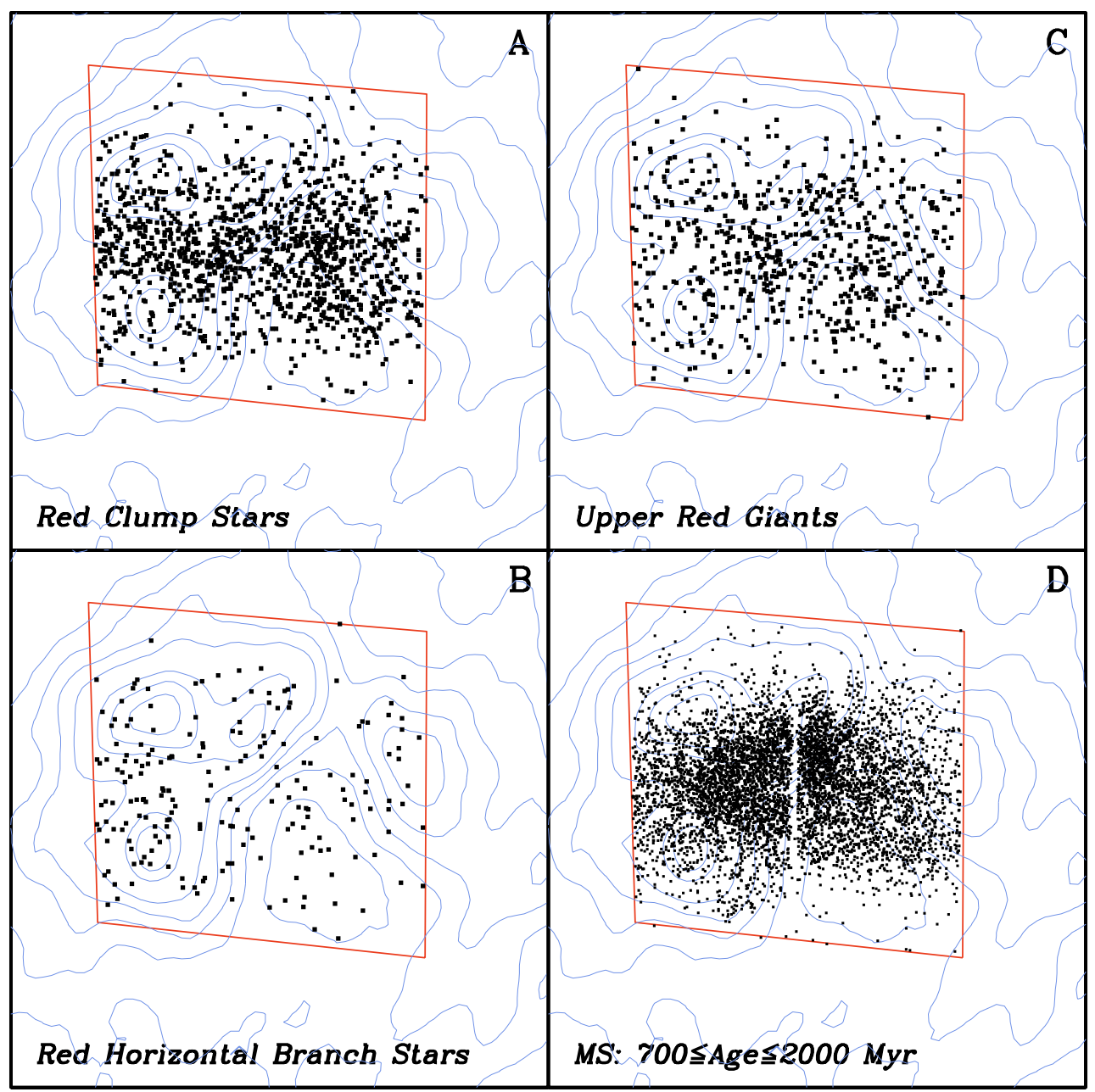

Fig. 12. Spatial distribution of relatively old stars. Panel A) shows the distribution of bright RC stars with $24.8 \leq m_{F 606 W} \leq 25.4$ and $0.5 \leq\left(m_{F 475 W}-m_{F 606 W}\right) \leq 0.7$, corresponding to an age interval between 0.3 and 2.0 Gyr. Panel B) shows the distribution of presumably old red-HB stars, having $25.6 \leq m_{F 606 W} \leq 25.8$ and $0.4 \leq\left(m_{F 475 W}-m_{F 606 W}\right) \leq 0.6$. Panel C) shows the distribution of RGB stars brighter than $m_{F 606 W}=24.5$. Red giant stars are at least 1 Gyr old. For comparison, Panel D) shows the distribution of MS stars with age between 0.7 and 2.0 Gyr $\left(26.5 \leq m_{F 606 W} \leq 27.5\right)$.

that should still be somewhere within the Hi hole (it is extremely unlikely that such clusters would have dispersed completely during the formation time of the hole). Because the stellar surface brightness within the H I hole in SagDIG is clearly very low we will make assumptions in this estimate so as to minimize the stellar luminosity which should remain.

From the H I properties of the H I hole in SagDIG we can estimate the energy required to create it from the expression given by Heiles (1979):

$E_{\mathrm{E}}=5.3 \times 10^{43} n_{0}^{1.12}\left(\frac{d}{2}\right)^{3.12} \sigma_{\mathrm{v}}^{1.4} \quad \mathrm{erg}$

where $d$ is the diameter of the hole in parsecs, $n_{0}$ is the hydrogen particle density in $\mathrm{cm}^{-3}$ and $\sigma_{\mathrm{v}}$ is the velocity dispersion of the atomic gas (in the case of SagDIG no expansion of the H I hole is detected). This is a lower limit if there is expansion in the plane of the galaxy which is hidden by the face-on orientation of SagDIG. The detailed HI observations of Young et al. have allowed all of these to be measured; they find, $d=1.3 \mathrm{kpc}, \sigma_{\mathrm{v}}=6-11 \mathrm{~km} \mathrm{~s}^{-1}$ and $n_{0} \simeq 0.22 \mathrm{~cm}^{-3}$ for the higher velocity dispersion component. Taking $\sigma_{\mathrm{v}}=10 \mathrm{~km} \mathrm{~s}^{-1}$ we find for the hole in the SagDIG $E_{\mathrm{E}} \sim 1.5 \times 10^{53} \mathrm{erg}$.

Assuming that a SN supplies $10^{51} \mathrm{erg}$ (McCray \& Kafatos 1987) of mechanical energy to the ISM this energy requirement implies that it was created by $\sim 150 \mathrm{SNe}$. Assuming a normal (Salpeter) IMF we therefore normalize the stellar mass distribution so that there are 150 stars more massive than $7 M_{\odot}$, which gives an IMF, $\Phi_{m}(m)=2880 m^{-2.35}$.

For this IMF the luminosity of all the stars less massive than $7 M_{\odot}$ will depend on the age of the population. Although this will fall with time the reduction in luminosity will be modest over the lifetime of the hole. For the moment we assume the hole has the same age as the lifetime of the lowest mass progenitor that undergoes a supernova, $\sim 5 \times 10^{7}$ yr (Oey \& Clarke 1997) but see below.

We estimate a lower limit to the luminosity of the remaining stars just after the last supernova occurred by assuming that 
they are all still on the main sequence. Using a standard main sequence luminosity function and integrating from 0.1 to $7 M_{\odot}$ we find a luminosity of $4500 L_{\odot}$. In order to reduce this number we must invoke aging of the population, but stellar evolution models show that between $5 \times 10^{7}$ and $10^{8}$ yr this amounts to less than a factor of 2.

At the distance of SagDIG $4500 L_{\odot}$ corresponds to $m_{\mathrm{v}} \simeq$ 21. Within the Hi hole we see no evidence of any clusters brighter than $m_{\mathrm{v}} \simeq 26.0$ and so if the star formation episode that formed the hole was confined to one or even several clusters these clusters must have completely dispersed on a timescale of $5 \times 10^{7}$ years. Perhaps the star formation that formed the hole was not confined to clusters but was more widespread? Although the total stellar light integrated within the H I hole does exceed 21 mag this light is distributed smoothly with an intensity that increases gradually towards the optical center of the galaxy. The surface brightness is always below that in other regions of the galaxy. For the supernova hypothesis to be feasible then, we require that the stars with ages in the approximate range 50-100 Myr are concentrated within the $\mathrm{H}$ I hole region of the galaxy. In Fig. 11 we see that the only stars in this age range within the $\mathrm{HI}$ hole are located at its NW edge and that there are more stars in this age range beyond the confines of the hole. Indeed there is no evidence of any age stellar population that is preferentially distributed within the $\mathrm{H}$ I hole.

Possible ways to reconcile the data with the supernovadriven wind hypothesis.

- The cluster or clusters in which the stars formed have been completely dispersed within $\sim 5 \times 10^{7} \mathrm{yr}$. For a stellar cluster to be so short-lived one would expect a dynamic disruption (such as the passage through a spiral arm or tidal interaction) to be the cause. Neither of these seem to be present in SagDIG and so one would expect clusters to be long lived.

- The hole is very old. As the Hi hole shows no evidence for expansion it could have formed some time in the past and just be a relic of an ancient star formation event. However, the velocity dispersion of the HI which is observed to be $10 \mathrm{~km} \mathrm{~s}^{-1}$ places a limit on how long structure can survive. In the case of the hole in SagDIG, with a radius of $650 \mathrm{pc}$ this is $\sim 6 \times 10^{7} \mathrm{yr}$. Therefore, adding the time of formation, the stars that formed coevally with those that expanded the hole should not be older than $\sim 10^{8}$ yr. Stellar evolution models show that for the luminosity of a cluster to fall by a factor of 100 (from mag 21 to our detection limit of 26) requires $\sim 2 \times 10^{9} \mathrm{yr}$. We cannot therefore reconcile our non-detection of clusters with the presence of the H I hole.

- A very top-heavy IMF. The analysis in first part of this paper gives no support for anything but a normal IMF in SagDIG.

- The energy imparted to the ISM by a supernova is roughly 2 orders of magnitude above the value we have used of $10^{51} \mathrm{erg}$, or the energy required to produce the hole is lower by a similar amount. Both of these would require that theories of supernovae be substantially revised.
In the case of SagDIG the depth of the ACS data allow us to conclude that the supernova driven wind hypothesis is an extremely unlikely explanation for the shell structure in SagDIG. Other authors have come to similar conclusions for the formation of H i holes (Crosthwaite et al. 2000; Deul \& den Hartog 1990; Rhode et al. 1999) but that in SagDIG is surely one of the most secure cases of a failure of the supernova wind hypothesis.

There are some possible alternatives to the supernovadriven wind hypothesis.

- One possibility is that the Hi hole region represents a region where the ISM is dominated by molecular rather than atomic gas. Although the presence of molecular gas may not necessarily result in a detection of $\mathrm{CO}$ emission $(\mathrm{CO}$ is a poor tracer of molecular gas in low metallicity environments, Taylor et al. 1998; Rubio et al. 2004) one would expect to see some evidence of recent star formation associated with the molecular gas. The absence of young stars in this region therefore argues against this interpretation.

- Another possibility is that the large gaseous ring is the result of a dynamical resonance in a non-axisymmetric potential. For such rings to form however the gas surface density must exceed the critical density given by the Toomre stability criterion. We cannot calculate this without knowledge of the rotation curve and so for SagDIG this cannot be tested. However, the gas surface density rarely exceeds the critical density in dwarf irregular galaxies in which the rotation curve has been measured (e.g. ESO215-G009, Warren et al. 2004).

- A mechanism that may apply to SagDIG and also to dwarfs containing a single large H I "hole" such as the Local Group galaxies Sextans A (van Dyk et al. 1998) and LeoA (Young \& Lo 1996) but probably not to dwarfs like Ho II which contain many smaller holes could simply be that the H I distribution was never uniform in the first place. The accretion of an intra-group H I cloud by a low mass dark matter halo (in which some star formation had taken place at earlier epochs) would result in a far from uniform H I distribution. The non-zero angular momentum of the cloud with respect to the halo would naturally lead to the formation of a ring of gas. The ring would form at the radius where the gravitational force balanced the orbital angular momentum. For atomic gas that has been recently acquired from an "external" source there is little reason to expect any correlation between the stars and the gas in the resulting system. Interaction between accreted gas and pre-existing ISM could have triggered the most recent star forming event (Fig. 11 panel A). The HI structure around the Phoenix dwarf mapped by Young \& Lo (1997) in which 2 large clouds of H I may be present, neither of which is coincident with the stellar distribution, is consistent with this scenario. Rhode et al. (1999) discuss several alternative explanations for the formation of large H I holes in Ho II, including gamma-ray bursts, impacts of high velocity clouds (HVCs), large scale turbulence and ionization by the intergalactic radiation field. These possibilities are all equally applicable to SagDIG. Apart from noting that SagDIG is very isolated 
and that there is no evidence of nearby H I clouds we can do little to test these ideas.

\section{Summary}

We have presented a study of the stellar populations in SagDIG to unprecedented deepness using observations obtained with the Advanced Camera for Surveys aboard HST. Our HST/ACS photometry is deep enough to go fainter than the mainsequence turnoff of halo, thick and thin disk stars in the Milky Way. Indeed, using ACS we can observe SagDIG looking through the Galaxy. Our deep color-magnitude diagrams reach down to $m_{F 606 W} \sim 27, m_{F 814 W} \sim 27$, which allowed us to observe the stellar populations of SagDIG to a surprising degree of detail. The main results are the following:

- As a result of the photometric precision of ACS and our choice of a wide color baseline, $m_{F 475 W}-m_{F 814 W}$, the young main-sequence is traced down to $m_{F 606 W} \sim 26$, well separated from the sequence of He burning blue super-giants on the blue-loop phase. This allowed us to estimate the mean star-formation rate in the last $0.6 \mathrm{Gyr}$.

- There is a well defined RGB, indicating that the dominant stellar population if older than $\sim 1$ Gyr. From the mean color of RGB stars we infer a mean stellar metallicity $[\mathrm{Fe} / \mathrm{H}]=$ -2.21 (uncorrected for age effects).

- Previously known carbon stars are easily identified on the CMD. Most of them belong to an extended AGB typical of an intermediate age population. However, a few $\mathrm{C}$ stars located with high precision below the tip of the RGB may belong to an old population.

- The distribution of He burning stars in the CMD is indicative of an extended period star formation, spanning a time interval between as early as $10 \mathrm{Gyr}$ ago and $100 \mathrm{Myr}$ ago. Most of the stars populate a very rich "red clump", which is the locus of stars older than $\sim 1$ Gyr. The stars younger than $1 \mathrm{Gyr}$ are more luminous than the $\mathrm{RC}$, evolving on the blue-loop phase to form the sequence of blue supergiants, and the associated (less prominent) sequence of red super-giants, contaminating the blue side of the RGB. The luminosity distribution of the blue super-giants will be used in a separate paper to reconstruct the history of star formation up to $1 \mathrm{Gyr}$ ago. The wide color range of blue-loop stars suggests that even the recently formed stars are substantially metal-poor, among the most metal-poor in dwarf irregular galaxies $(Z \approx 0.0004)$.

- The presence of a red HB, noticed as a fainter extension of the RC - proves that an early epoch of star formation took place in this dwarf irregular galaxy. This result is of foremost importance since it sets a lower limit to the first epoch of star formation in yet another actively star-forming dwarf galaxy. In this regard, the resulting scenario is one in which all Local Group galaxies show to have had an ancient ( $\sim 10$ Gyr) initial episode of star formation. The only one exception to this result is IZw 18 (Izotov \& Thuan 2004) located at $\sim 15 \mathrm{Mpc}$ and believed to be relatively young ( $\leq 2 \mathrm{Gyr}$ ). In the appendix we briefly comment on this.
- We have discussed the spatial distribution of stars in different age intervals. We find the distribution of young stars to be clumpy and associated with the regions of high emission from the neutral gas, but not coincident with the maxima of H I column density. Older main-sequence stars (with age between 0.7 and $2 \mathrm{Gyr}$ ) and intermediate-age (RC and RGB) stars show an extended, although not perfectly uniform, distribution. There is no obvious correlation between the distribution of these relatively old stars and that of the neutral gas.

- By considering the energy input from supernova explosions, we find that the "hole" in the H I distribution cannot have been produced by a supernova driven wind unless, 1) the hole is unfeasibly old; 2) supernovae are able to impart 2 orders of magnitude more kinetic energy to the ISM than previously thought; or 3) the IMF is very top-heavy. We can reasonably exclude 1 and 3 , and 2 is very unlikely. By far the most difficult obstacle for the supernova wind driven hypothesis is the fact that the stellar surface brightness within the $\mathrm{H}$ I hole is lower than that outside the hole for any stellar age range. We find an appealing alternative to be that the H I ring (rather than "hole") is the result of a recent accretion of an intra-group $\mathrm{H}$ I cloud with a non-zero angular momentum.

Acknowledgements. We thank the anonymous referee for comments that improved the presentation of the paper. We also thank Dr. L. Young for kindly providing us with her Hi density maps, Dr. L. Girardi for making available to us his set of theoretical isochrones on the ACS system, Dr. E. Brinks for helpful comments on the manuscript, and Dr. M. Sirianni for communicating his results on ACS characterization in advance of publication. E.V.H. wishes to thank the European Southern Observatory for hospitality during a visit in which this paper was partly written. Y.M. acknowledges financial support by MIUR under the program PRIN2003.

\section{Appendix A: On the age of $I Z w 18$}

The discovery of a population of presumably very old stars (red HB stars) in SagDIG argues against metal-poor galaxies being necessarily young, i.e. born in recent times. In this regard, we found it of interest to re-examine the properties of the prototype of candidate young galaxies, I Zw 18 .

When comparing the two galaxies, it should be remembered that the metallicity difference between the two galaxies is not large: IZw 18 is not so different after all from the most metal-poor Local Group dwarf galaxies like SagDIG or Leo A (see Aloisi et al. (2003) for a recent compilation of heavy elements abundances in IZw 18). Yet IZw 18 is a key object in that it lacks a positive detection of a red giant branch, which seems to imply that the galaxy is younger than about 1 Gyr. Even the recent HST/ACS deep study of Izotov \& Thuan (2004) was unable to trace an "old" RGB. These authors place IZw 18 further out than previously thought, at $15 \mathrm{Mpc}$ instead of $10 \mathrm{Mpc}$, and set an upper limit to the age of IZw 18 between 1-2 Gyr.

By applying the reduction methods described in Sect. 2 to the ACS data of Izotov \& Thuan (2004), we have been able to obtain a substantially improved CMD of IZw 18 . The result of 


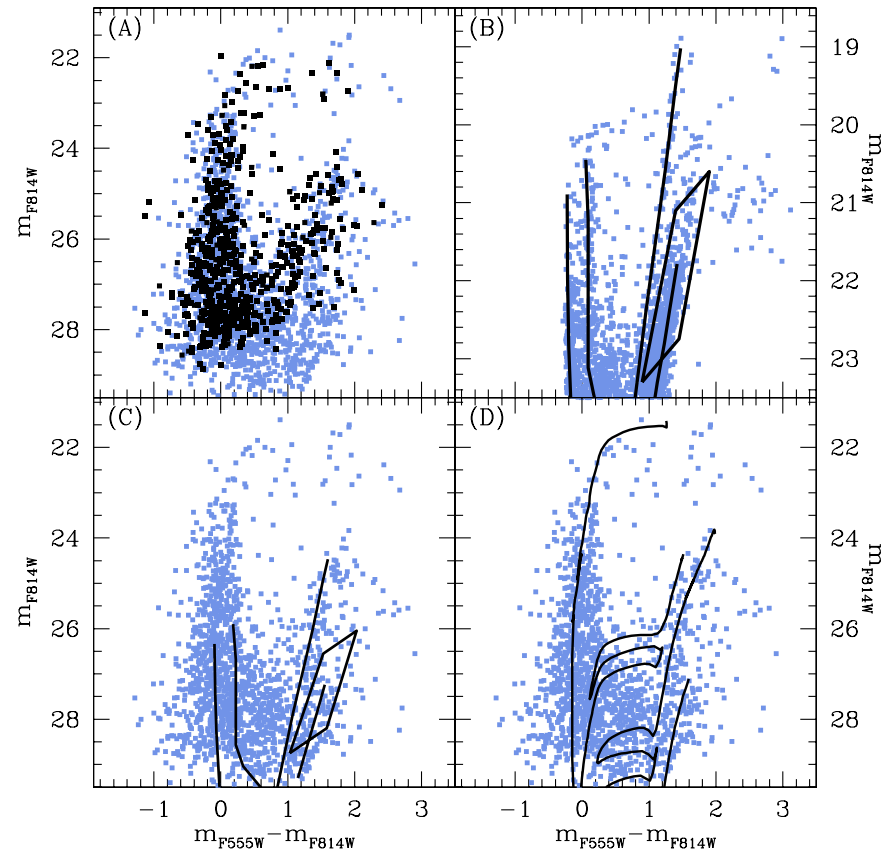

Fig. A.1. The $m_{F 814 W},\left(m_{F 555 W}-m_{F 814 W}\right)$ color-magnitude diagram of IZw 18 from ACS archival data. Panel A) compares the photometry of Izotov \& Thuan (2004, dark dots) with the result of our reanalysis (light dots), showing a better recovery of the fainter red stars. The HST/WFPC2 color-magnitude diagram of Sextans A (Dolphin et al. 2003) is shown for comparison in Panel B), together with handdrawn fiducial lines marking the major evolutionary sequences (MS, blue and red super-giants, and RGB). A box delimits red giant stars in the RGB tip region. The same fiducial loci are superposed to the CMD of IZw 18 in Panel C), corrected for the distance and reddening of Sextans A and shifted to the distance of IZw 18, assuming $(m-M)_{0}=30.88(15 \mathrm{Mpc})$ with $E(B-V)=0.15$. On the same assumptions, theoretical isochrones from Girardi et al. (2004, priv. comm.) with $Z=0.0004$ and ages 7, 80, $281 \mathrm{Myr}$ and $10 \mathrm{Gyr}$ are over-plotted in Panel D).

our reanalysis is presented in Fig. A.1, where we show a new $m_{F 814 W},\left(m_{F 555 W}-m_{F 814 W}\right)$ CMD for a combined field containing both the main body and the "C" component of I Zw 18.

The new diagram is shown in Panel A, along with that of Izotov \& Thuan (2004). The comparison indicates an excellent match for the CMD features in common, with no appreciable photometric differences between the two reductions. Our reduction however provides access to a higher completeness everywhere, and deeper photometry. This result is probably to be attributed to the updated geometrical distortion correction and pixel oversampling technique employed here (see Anderson \& King 2000) which appears to improve the effectiveness of PSF fitting. Note that the selection criteria on ALLSTAR parameters are similar for both reductions.

It is for the redder population that the improvements in our diagram are most noticeable. In particular, we can identify with some confidence the sequence of red stars having colors $\left(m_{F 555 W}-m_{F 814 W}\right)=1-2$, made of red super-giants. This identification, crucial to set observational constraints on the reconstruction of the SFH of IZw 18, is supported by comparison with the CMDs of other dwarf irregular galax- ies. Panel B shows the HST/WFPC2 color-magnitude diagram of Sextans A obtained by Dolphin et al. (2003), where we have marked the main features (MS, blue and red super-giants, and RGB) with fiducial lines. The same fiducial loci are superimposed onto the CMD of IZw 18 in Panel C, assuming a distance of $15 \mathrm{Mpc}$ for the galaxy. While the higher luminosity reached by MS stars in IZw 18 is consistent with its stronger star-burst, this comparison shows an intriguing agreement for the older evolutionary sequences, in particular around the red super-giant and RGB tip regions. This conclusion is confirmed by a comparison with isochrones from Girardi et al. with $Z=0.0004$ and ages 7, 80, $281 \mathrm{Myr}$, and $10 \mathrm{Gyr}$, which are over-plotted on the data in Panel D.

The termination point of the $10 \mathrm{Gyr}$ isochrone overlaps with the clump of stars seen just fainter than $m_{F 814 W} \sim 27.3$. Note also that the magnitude difference between the tip of the old RGB and that of the RSG is typical of dwarf irregulars, spanning 3.5-4.5 mag in $I$ with some dependence on the SFH of each galaxy. Although this result does not yet represent a strong case for the identification of an "old" RGB, it seems very encouraging. Our reanalysis gives supports to the $15 \mathrm{Mpc}$ distance proposed by Izotov \& Thuan (2004), and provides preliminary yet intriguing evidence for a RGB cutoff at $m_{F 814 W} \sim 27.3$. In this scenario, IZw 18 would not appear substantially different from extremely metal-poor (yet old) Local Group dwarfs like SagDIG and Leo A. Efforts to push even deeper the capabilities of ACS would be worthwhile to confirm this hypothesis.

\section{References}

Aloisi, A., Tosi, M., \& Greggio, L. 1999, AJ, 118, 302

Aloisi, A., Savaglio, S., Heckman, T., et al. 2003, ApJ, 595, 760

Aparicio, A., Bertelli, G., \& Chiosi, C. 2000, in preparation

Anderson, J., \& King, I. R. 2000, PASP, 112, 1360

Baldacci, L., Matonti, F., Rizzi, L., et al. 2004, Mem. Soc. Astron. It., 75, 126

Bedin, L. R., Cassisi, S., Castelli, F., et al. 2005, MNRAS, 357, 1038

Bellazzini, M., Ferraro, F. R., \& Pancino, E. 2001, ApJ, 556, 635

Brown, T., Ferguson, H., Smith, E., et al. 2003, ApJ, 592, L17

Brown, T., Ferguson, H., Smith, E., et al. 2004, AJ, 127, 2738

Chiosi, C., Bertelli, G., \& Bressan, A. 1992, ARA\&A, 30, 235

Cook, K. H. 1987, Ph.D Thesis, Univ. Arizona (C87)

Crosthwaite, L. P., Turner, J. L., \& Ho, P. T. P. 2000, AJ, 119, 1720

Da Costa, G. S., \& Armandroff, T. E. 1990, AJ, 100, 162

Demers, S., \& Battinelli, P. 2002, AJ, 123, 238

Deul, E. R., \& den Hartog, R. H. 1990, A\&A, 229, 362

Dohm-Palmer, R. C., Skillman, E. D., Saha, A., et al. 1997, AJ, 114, 2527

Dohm-Palmer, R. C., Skillman, E. D., Gallagher, J., et al. 1998, AJ, 116,1227

Dohm-Palmer, R. C., Skillman, E. D., Mateo, M., et al. 2002, AJ, 123, 813

Dolphin, A. E. 2000, ApJ, 531, 804

Dolphin, A. E., Saha, A., Skillman, E. D., et al. 2001, ApJ, 550, 554

Dolphin, A. E., Saha, A., Claver, J., et al. 2002, AJ, 123, 3154

Dolphin, A. E., Saha, A., Skillman, E. D., et al. 2003, AJ, 126, 187

Downes, R. A., Margon, B., Anderson, S. F., et al. 2004, AJ, 127, 2838

Girardi, L., Bertelli, G., Bressan, et al. 2002, A\&A, 391, 195

Harris, W. E. 1996, AJ, 112, 1487 
Heiles, C. 1979, ApJ, 229, 533

Holtzman, J. A., Hester, J. J., Casertano, S., et al. 1995, PASP, 107, 156

Izotov, Y. I., \& Thuan, T. X. 2004, ApJ, 616, 768

Karachentsev, I., Aparicio, A., \& Makarova, L. 1999, A\&A, 352, 363

Krist, J. 2003, Instrument Science Report ACS 2003-06

Lee, M. G., Freedman, W. L., \& Madore, B. F. 1993, ApJ, 417, 553

Lee, M. G., \& Kim, S. C. 2000, AJ, 119, 777

Lo, K. Y., Sargent, W. L., \& Young, K. 1993, AJ, 106, 507

Mateo, M. 1998, ARA\&A, 36, 435

McCray, R., Shull, J. M., \& Sutherland, P. 1987, ApJ, 317, L73

Momany, Y., Held, E., Saviane, I., \& Rizzi 2002, A\&A, 384, 393 (M02)

Oey, M. S., \& Clarke, C. J. 1997, MNRAS, 289, 570

Pietrinferni, A., Cassisi, S., Salaris, \& Castelli 2004, ApJ, 612, 168

Rhode, K., Salzer, Westpfahl, D., \& Radice, L. 1999, AJ, 118, 323

Rizzi, L., Held, E. V., Bertelli, G., \& Saviane, I. 2003, ApJ, 589, L85

Robin, A. C., Reylé, C., Derrière, S., \& Picaud, S. 2003, A\&A, 409, 523

Rubio, M., Boulanger, \& Contursi, A. 2004, A\&A, 425, L1
Saviane, I., Held, E. V., \& Bertelli, G. 2000, A\&A, 355, 56

Saviane, I., Rizzi, L., Held, E., Bresolin, F., \& Momany, Y. 2002, A\&A, 390, 59 (S02)

Saviane, I., Hibbard, J. E., \& Rich, R. M. 2004, AJ, 127, 660

Schlegel, D. J., Finkbeiner, D. P., \& Davis, M. 1998, ApJ, 500, 525

Searle, L., \& Sargent, W. 1972, ApJ, 173, 25

Skillman, E. D., Terlevich, R., \& Melnick, J. 1989, MNRAS, 240, 563

Skillman, E., Tolstoy, E., Cole, A., et al. 2003, ApJ, 596, 253

Sirianni, M., Jee, M. J., Benítez, N., et al. 2004, preprint Stetson, P. B. 1987, PASP, 99, 191

Stewart, S. G., Fanelli, M. N., Byrd, G. G., et al. 2000, ApJ, 529, 201

Strobel, N. V., Hodge, P., \& Kennicutt, R. C. J. 1991, AJ, 383, 148

Taylor, C., Kobulnicky, H., \& Skillman, E. 1998, AJ, 116, 2746

Tolstoy, E., Gallagher, J., Cole, A. A., et al. 1998, AJ, 116, 1244

van Dyk, S. D., Puche, D., \& Wong, T. 1998, AJ, 116, 234

Warren, B. E., Jerjen, H., \& Koribalski, B. S. 2004, AJ, 128, 1152

Westera, P., Lejeune, T., Buser, R., Cuisinier, F., \& Bruzual, G. 2002, A\&A, 381, 524

Young, L. M., \& Lo, K. Y. 1996, ApJ, 462, 203

Young, L. M., \& Lo, K. Y. 1997, ApJ, 490, 710 